\title{
A RADIAL VELOCITY SURVEY OF THE CYG OB2 ASSOCIATION
}

\author{
Daniel C. Kiminki, ${ }^{1}$ Henry A. Kobulnicky, ${ }^{1}$ K. Kinemuchi, ${ }^{1}$ Jennifer S. Irwin, ${ }^{2}$ Christopher L. Fryer, ${ }^{3,4}$ \\ R. C. Berrington, ${ }^{1}$ B. Uzpen, ${ }^{1}$ Andy J. Monson, ${ }^{1}$ Michael J. Pierce, ${ }^{1}$ and S. E. Woosley ${ }^{5}$ \\ Received 2006 July 7; accepted 2006 September 27
}

\begin{abstract}
We conducted a radial velocity survey of the Cyg OB2 association over a 6 yr (1999-2005) time interval to search for massive close binaries. During this time we obtained 1139 spectra on $146 \mathrm{OB}$ stars to measure mean systemic radial velocities and radial velocity variations. We spectroscopically identify 73 new OB stars for the first time, the majority of which are likely to be association members. Spectroscopic evidence is also presented for a B3 Iae classification and temperature class variation (B3-B8) on the order of $1 \mathrm{yr}$ for Cyg OB2 No. 12. Calculations of the initial mass function with the current spectroscopic sample yield $\Gamma=-2.2 \pm 0.1$. Of the 120 stars with the most reliable data, 36 are probable and 9 are possible single-lined spectroscopic binaries. We also identify three new and eight candidate double-lined spectroscopic binaries. These data imply a lower limit on the massive binary fraction of $30 \%-42 \%$. The calculated velocity dispersion for Cyg OB2 is $2.44 \pm 0.07 \mathrm{~km} \mathrm{~s}^{-1}$, which is typical of open clusters. No runaway OB stars were found.
\end{abstract}

Subject headings: binaries: close — binaries: general — binaries: spectroscopic — stars: early-type — stars: kinematics - surveys - techniques: radial velocities

Online material: machine-readable tables

\section{INTRODUCTION}

Cyg OB2 may be one of the most massive and richest associations in the Galaxy with $2600 \pm 400 \mathrm{OB}$ cluster members (Knödlseder 2000, hereafter K00) and 90-100 O stars (Comerón et al. 2002; K00). It has been studied numerous times due to its richness, proximity (1.7 kpc; Hanson 2003; Massey \& Thompson 1991, hereafter MT91; Schulte 1958; Johnson \& Morgan 1954), and high-extinction components with $A_{V}>5$. The total mass of the cluster is estimated to be $(4-10) \times 10^{4} M_{\odot}$ with a central stellar density of $\rho_{0}=40-150 M_{\odot} \mathrm{pc}^{-3}(\mathrm{~K} 00)$. The radius of Cyg OB2 has been estimated as large as $30 \mathrm{pc}$ (K00). For the purposes of this study, we adopt a cluster core radius of $\sim 15 \mathrm{pc}$ $\left(30^{\prime}\right)$ inferred from the photometric survey of MT91.

A handful of massive binaries have been discovered in the association. Among the OB-type binaries are MT 421, MT 429, MT 506, MT 554, MT 696, Cyg OB2 No. 5, and Cyg OB2 No. 8a (MT 465). The first four of these are eclipsing binaries of the Algol type (Pigulski \& Kolaczkowski 1998; Kazarovets et al. 2000). Rios \& DeGioia-Eastwood (2004) discovered that MT 696 is an early contact binary (W UMa type) consisting of a late $\mathrm{O}$ and an early $\mathrm{B}$ star with a period of 1.46 days. Cyg OB2 No. 5 is possibly a triple system consisting of an O7 Ianfp+ Ofpe/WN9 contact binary with a period of 6.6 days (Leung \& Schneider 1978; Hall 1974) and a B0 V star (Benaglia et al. 2001; Walborn 1973; Bohannan \& Conti 1976). Cyg OB2 No. 8a is a nonthermal radio emitter (Bieging et al. 1989) and massive binary system consisting of O6 and O5.5 stars with a period of $\sim 22$ days (De Becker et al. 2004).

\footnotetext{
1 Department of Physics and Astronomy, University of Wyoming, Laramie, WY 82070

2 Department of Astronomy and McDonald Observatory, University of Texas, Austin, TX 78712.

3 Theoretical Astrophysics, Los Alamos National Laboratories, Los Alamos, NM 87545.

4 Department of Physics, University of Arizona, Tucson, AZ 85721.

5 Department of Astronomy and Astrophysics, University of California, Santa Cruz, CA 95064
}

Massive close binaries (MCBs) are the progenitors of some classes of energetic phenomena such as supernovae, $\gamma$-ray bursts, and X-ray binaries (Fryer et al. 1998, 1999). They are also laboratories in which to study the formation mechanisms for massive stars (Bonnell et al. 1998). Direct and indirect evidence reveals that up to $50 \%-80 \%$ of massive stars reside in binary systems (Vanbeveren 2004; Gies 1987; Garcia \& Mermilliod 2001). Studies of the MCB frequency are numerous and include Hillwig et al. (2006), Vanbeveren (2004), Zinnecker (2003), Garcia \& Mermilliod (2001), Bonnell et al. (1998), van der Woerd \& van den Heuvel (1984), and Garmany et al. (1980). Results have been all but conclusive. The binary frequency for Galactic B0-B3 stars is at least $32 \%$, and it may be anywhere between $14 \%$ and $80 \%$ for OB stars in general (Vanbeveren 2004; Vanbeveren et al. 1998; Garcia \& Mermilliod 2001). In addition, Vanbeveren (2004) speculates that there may not be a standard MCB frequency for open clusters and associations. The longterm goal of this study is to provide a measurement of the MCB frequency, binary star mass ratios, and orbital separations of Cyg OB2 stars.

Section 2 presents our observations and data reduction. Section 3 discusses the identification of new early-type stars and our derivations of cluster quantities such as the initial mass function (IMF), visual extinction, and distance. Section 4 describes the calculation of radial velocities. Section 5 presents the results and a measurement of the cluster velocity dispersion. Section 6 summarizes the survey findings.

\section{OBSERVATIONS}

Between 1999 July and 2005 October we observed 183 stars in the direction of the Cyg OB2 association with the echelle spectrographs at the Lick $3 \mathrm{~m}$ and $\mathrm{Keck}^{6} 10 \mathrm{~m}$ telescopes, the

\footnotetext{
6 The W. M. Keck Observatory is operated as a scientific partnership among the California Institute of Technology, the University of California, and the National Aeronautics and Space Administration. The Observatory was made possible by the generous financial support of the W. M. Keck Foundation.
} 
TABLE 1

OBSERVING INFORMATION

\begin{tabular}{|c|c|c|c|}
\hline Date & Observatory/Instrument & Spectral Coverage & HJD Coverage \\
\hline 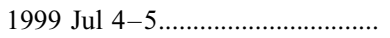 & Keck/HIRES & $3890-6270 \AA$ in 35 orders & $2,451,363-2,451,364$ \\
\hline 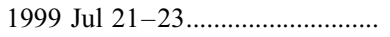 & Lick/Hamilton & $3650-7675 \AA$ in 81 orders & $2,451,381-2,451,383$ \\
\hline 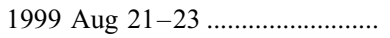 & Lick/Hamilton & $3650-7675 \AA$ in 81 orders & $2,451,411-2,451,413$ \\
\hline 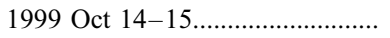 & Keck/HIRES & $3700-5250 \AA$ in 29 orders & $2,451,466-2,451,467$ \\
\hline 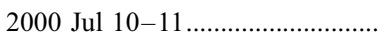 & Lick/Hamilton & $3650-7675 \AA$ in 81 orders & $2,451,736-2,451,737$ \\
\hline 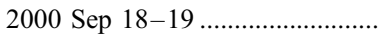 & Keck/HIRES & $3700-5250 \AA$ in 29 orders & $2,451,805-2,451,806$ \\
\hline 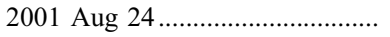 & WIYN/Hydra & $3800-4490 \AA$ in order 2 & $2,452,146$ \\
\hline 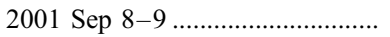 & WIYN/Hydra & $3800-4490 \AA$ in order 2 & $2,452,161-2,452,162$ \\
\hline 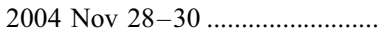 & WIYN/Hydra & $3800-4490 \AA$ in order 2 & $2,453,338-2,453,340$ \\
\hline 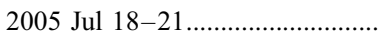 & WIRO/WIRO-Spec & $3800-4490 \AA$ in order 1 & $2,453,570-2,453,573$ \\
\hline 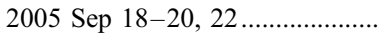 & WIRO/WIRO-Spec & $3800-4490 \AA$ in order 1 & $2,453,632-2,453,635$ \\
\hline 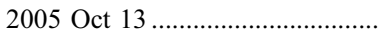 & WIRO/Longslit & $4050-6050 \AA$ in order 2 & $2,453,657$ \\
\hline
\end{tabular}

Hydra spectrograph at the WIYN ${ }^{7} 3.5 \mathrm{~m}$ telescope, the Wyoming Infrared Observatory (WIRO) fiber-bundle spectrograph (WIROSpec; Nations \& Pierce 2002), and the WIRO-Longslit spectrograph at the WIRO $2.3 \mathrm{~m}$ telescope. Table 1 lists the observing runs at each facility and the approximate spectral coverages. Stars were selected from the $U B V$ photometric and spectroscopic survey of Cyg OB2 by MT91. We included 73 stars with previous spectroscopic OB classification from Table 5 of MT91. During the initial 1999 Keck run we included 110 additional stars from MT91 having reddening-free parameter $Q \equiv(U-B)-$ $0.8(B-V)$ corresponding to stars earlier than $\sim \mathrm{B} 3$. Our motivation was to obtain a more complete sample of reddened earlytype stars. However, this more inclusive sample necessarily contained foreground $\mathrm{A}-\mathrm{G}$ stars and background or foreground OB stars.

At the Lick $3 \mathrm{~m}$ telescope, the Hamilton echelle spectrograph was used to cover the wavelength range $3620-7675 \AA$ in 82 spectral orders with a mean spectral resolution of $R \sim 30,000$. These observations occurred over eight nights, 1999 July 2123, 1999 August 21-23, and 2000 July 10-11. Exposure times ranged between 240 and $1200 \mathrm{~s}$. Hourly exposures of a thoriumargon arc lamp served to calibrate the wavelength of each exposure to an rms of $0.002 \AA\left(0.12 \mathrm{~km} \mathrm{~s}^{-1}\right.$ at $\left.5000 \AA\right)$. The typical resolution was $0.12 \AA$ FWHM at $5700 \AA$ and $0.08 \AA$ FWHM at $3700 \AA$. A $1.2^{\prime \prime} \times 2.5^{\prime \prime}$ slit decker was used throughout. Observations of six radial velocity standard stars were used to confirm the repeatability of the wavelength calibration from epoch to epoch.

We acquired spectra at Keck with the HIRES spectrograph (Vogt et al. 1994) on 1999 July 4-5, 1999 October 14-15, and 2000 September 18-19. We used the blue collimator to obtain $R \sim 30,000$ spectra over the wavelength range $3600-5200 \AA$ (3900-5800 A on the 1999 July 4-5 run). On the 1999 July run, spectral regions from 5163 to $5172 \AA$ and from 5238 to $5246 \AA$ are unusable due to blemishes on the detector, while on the other Keck runs, spectral regions from 4512 to $4517 \AA$ are unusable. The slit decker $\mathrm{C} 5$ measuring $1.15^{\prime \prime}$ in the spectral direction and $7.0^{\prime \prime}$ in the spatial direction was used throughout. Periodic exposures of a thorium-argon arc lamp were used to calibrate the radial velocity of the spectra, which were then tied to radial velocity standards. The wavelength scale of each exposure is good to an rms of $0.003 \AA\left(0.18 \mathrm{~km} \mathrm{~s}^{-1}\right.$ at $\left.5000 \AA\right)$. The output

\footnotetext{
${ }^{7}$ The WIYN Observatory is a joint facility of the University of WisconsinMadison, Indiana University, Yale University, and the National Optical Astronomy Observatory.
}

pixel scale is $\sim 0.04 \AA$ pixel $^{-1}$. The mean instrumental resolution was $0.12 \AA \mathrm{FWHM}$ at $5000 \AA$ and $0.09 \AA \mathrm{FWHM}$ at $3700 \AA ̊$.

At WIYN we used the Hydra spectrograph with the Red camera, $2^{\prime \prime}$ blue fibers, and the 1200 line $\mathrm{mm}^{-1}$ grating in second order to obtain three $1200 \mathrm{~s}$ exposures in each of three configurations ( $\sim 90$ stars each). The spectral coverage was $3800-4500 \AA$ at a mean resolution of $R \sim 4500$. Observations at WIYN took place over six nights, 2001 August 24, 2001 September 8-9, and 2004 November 28-30. Helium-neonargon lamps were used between each exposure to calibrate the spectra to an rms of $0.03 \AA\left(2 \mathrm{~km} \mathrm{~s}^{-1}\right.$ at $\left.4500 \AA\right)$, and the typical resolution was $1.0 \AA \mathrm{FWHM}$ at $3900 \AA$ and $0.82 \AA \mathrm{FWHM}$ at $4400 \AA$.

At WIRO we used the WIRO-Spec fiber bundle spectrograph with $15 \times 20$ fiber array and $1^{\prime \prime}$ fibers to achieve $R \sim 4500$ over the wavelength range $4075-4910 \AA$ at a dispersion of $0.41 \AA$ pixel $^{-1}$. Observations at WIRO occurred over eight nights, 2005 July 18 21 and September 18-20 and 22. Copper-argon lamp exposures were used every 30 minutes to wavelength-calibrate the spectra to an rms of $0.1-0.2 \AA\left(6.7-13 \mathrm{~km} \mathrm{~s}^{-1}\right.$ at $\left.4500 \AA\right)$. The mean spectral resolution was $0.98 \AA \mathrm{FWHM}$ at $4500 \AA$. Typically, three to five $600 \mathrm{~s}$ exposures were obtained for each object. At WIRO, only a handful of the brightest stars with previously discovered large-amplitude velocity variations were observed.

We also obtained spectra of the seven brightest stars exhibiting large velocity variations with the WIRO-Longslit spectrograph on 2005 October 13 . We used a $2.5^{\prime \prime}$ slit width. The spectra covered the wavelength range 3950-6050 $\AA$ at a dispersion of $1.12 \AA$ pixel $^{-1}$. Argon lamps were used to calibrate the spectra to an rms of $0.15 \AA\left(10 \mathrm{~km} \mathrm{~s}^{-1}\right.$ at $\left.4500 \AA\right)$. The mean spectral resolution was $3.2 \AA$ FWHM at $4500 \AA$. Two or three $600 \mathrm{~s}$ exposures were acquired for each object.

The data were reduced in IRAF using standard data reduction techniques that included flat-fielding with exposures of an internal or dome quartz continuum lamp. One-dimensional spectra were extracted and wavelength-calibrated by interpolating a wavelength solution determined from periodic exposures of the arc lamps throughout the night. At each observatory and instrument, the variation in the wavelength solution during the night was small $(<0.5 \AA)$, monotonic, and well constrained by the frequent arc calibration. Because many of the nights were not photometric, no attempt at flux calibration was made. The one-dimensional spectra were corrected to a heliocentric velocity scale by computing the appropriate Doppler correction for each source, date, time of observation, and observatory using 
IRAF tasks rvcor and dopcor. Similar spectra from a given night were then combined, weighted by the signal-to-noise ratio $(\mathrm{S} / \mathrm{N})$. The final $\mathrm{S} / \mathrm{Ns}$ vary with wavelength, magnitude, telescope, instrument, and observing run. They range from 150:1 per pixel near $4400 \AA$ for WIYN observations of the brightest stars to $5: 1$ for some of the Lick data where intermittent clouds affected the data.

Observations of the radial velocity standard stars HD 012929 (spectral type K2 III), HD 171391 (G8 III), HD 182572 (G8 IV), HD 187691 (F8 V), HR 1320 (B2 IV), and HD 1174 (B3 V) were obtained on the Lick and Keck nights as a check on the wavelength calibration. Inspection of the corrected radial velocity standard star spectra shows excellent agreement between all epochs, giving confidence that the data are free from systematic velocity offsets between epochs and telescopes. The epoch-toepoch velocity dispersion among velocity standards after correction to the heliocentric reference frame is $0.05 \AA\left(\sim 3 \mathrm{~km} \mathrm{~s}^{-1}\right)$, i.e., less than the instrumental resolution.

The current data set includes 1139 individual spectra obtained over 29 epochs for 183 stars, 146 of which are OB stars. The mean number of observations per star was 7 , the median was 6 , the minimum was 1 , and the maximum was 19 .

\section{NEW SPECTRAL CLASSIFICATIONS AND DERIVED QUANTITIES}

We estimated new spectral types for all stars by visual comparison with the stellar atlas of Walborn \& Fitzpatrick (1990). Table 2 provides a list of all OB stars in our survey using the nomenclature of MT91. Columns (2) and (3) list spectral types from the literature for each object (primarily from MT91; see their Table 5) and as determined by our survey, respectively. Spectral types were determined independently by two authors, with the exception of Cyg OB2 No. 12, and there was generally good agreement to within one spectral class. Of the 110 stars without previous spectral classifications, 73 were OB stars, while 37 were A or later. These 37 non-OB stars are listed in Table 3 and range from $A$ to $M$.

\subsection{Cyg OB2 No. 21}

Two thoroughly observed stars, Cyg OB2 No. 12 and No. 21, exhibited large discrepancies between our new spectral type and those listed in the literature. Torres-Dodgen et al. (1991) spectroscopically classify Cyg OB2 No. 21 (MT 259) as a B1 and photometrically as B1 V. MT91 also classified this star a B1 V. Spectra of all epochs obtained on MT 259 were consistent, and we find that this star is best represented by a B0 Ib spectral classification. In Figure 1 we present a spectrum of MT 259 taken on 2001 September 9 at the WIYN observatory. The most distinguishing feature of this star's spectrum is the large number of metal lines, especially around $\mathrm{H} \delta \lambda 4101$. Figure 1 also shows the spectra of HD 164402 (B0 Ib) and HD 144470 (B1 V) from the Walborn \& Fitzpatrick (1990) spectral atlas for comparison.

\subsection{Cyg OB2 No. 12}

Cygnus OB2 No. 12 (MT 304) is one of the most notable stars in the association. It holds the reputation as one of the most luminous and reddened objects in the Galaxy (Sharpless 1957; Johnson 1968). It is also a bright X-ray source (Harnden et al. 1979) and variable radio emitter (Setia Gunawan et al. 2003). The X-ray emission is best explained by wind-driven shocks (Waldron et al. 1998, 2004), where No. 12 has a measured high stellar wind of $V_{\text {wind }}=1400 \mathrm{~km} \mathrm{~s}^{-1}$ (Leitherer \& Wolf 1982; Bieging et al. 1989) and an expanding shell of $V=3100 \mathrm{~km} \mathrm{~s}^{-1}$ (Wang \& Zhu 2003). It has been shown to vary photometrically
(Gottleib \& Liller 1978; Voelcker 1975), and Souza \& Lutz (1980) submit that a binary companion may provide an explanation for this. MT91 suggest the possibility that No. 12 is a luminous blue variable because it appears to vary spectroscopically as well as photometrically and has an extremely high luminosity.

Most studies adopt a spectral type for No. 12 of B5 Iae (MT91) or B8 Iae (Souza \& Lutz 1980). Abbott et al. (1981) adopt B3 Iae, but little justification for such an early classification is found in the literature. We find evidence for a B3 Iae spectral type and a temperature class variation between B3 and $\mathrm{B} 8$ by comparison with the OB star atlas of Walborn \& Fitzpatrick (1990), the supergiant atlas of Lennon et al. (1992), and the spectral atlas of Yamashita \& Nariai (1977). Figure 2 presents segments of the spectrum obtained in 2000 September at Keck. The top left panel shows the temperature-sensitive ratio He I $\lambda 4471 / \mathrm{Mg}$ II $\lambda 4481$, the strongest evidence for a B3 Iae spectral type. $\mathrm{Mg}$ II is a weaker feature than He I for temperature classes earlier than B7 and is stronger than $\mathrm{He}$ I for a B8 class or later. The observed ratio is approximately $2: 1$, characteristic of a B3 temperature class. It should be noted that the spectrum presented by Souza \& Lutz (1980) shows a ratio characteristic of a B8 class (Souza \& Lutz 1980, Fig. 1), and the spectrum presented by MT91 shows a ratio characteristic of B3 (although it is reported as B5 due to additional arguments; see Fig. 12 in MT91). The presence of the absorption feature $\lambda 4542$ (top right panel) provides additional evidence for a B3 classification. The relative intensity and asymmetric profile of the absorption suggest a blend of the two lines He II $\lambda 4542$ and Fe II $\lambda 4542$. This is generally only seen in the earliest of B stars. The bottom left panel shows $\mathrm{H} \beta \lambda 4861, \mathrm{He}$ I $\lambda 4922$, and a weak Fe II $\lambda 4923$. The strength of the He I absorption in relation to the weak Fe II absorption suggests an earlier type. The bottom right panel shows an example of deep absorption in one of the higher ionization lines, $\mathrm{N}$ III $\lambda 4097$. There is also a possible He II feature at $4100 \AA$, next to $\mathrm{H} \delta \lambda 4101$. We see additional evidence for weak He II absorption toward the blue end of the spectrum (i.e., 3710 and $3720 \AA$ ). The absence of Si IV $\lambda 4089$ suggests a classification no earlier than B3. The top left and bottom right panels also display clear examples of documented emission-line cores in No. 12 (He I 24471 and N III 24097 ).

Fluctuations in the spectrum of No. 12 are seen in our 2001 August and September spectra obtained at WIYN and are shown in Figure 3. Souza \& Lutz (1980) noted small spectral fluctuations in their spectra over a timescale of days. Figure 3 shows three of our spectra obtained over the span of $1 \mathrm{yr}$ and demonstrates a temperature class evolution between B3 and B8. The upper spectrum is from Keck HIRES on 2000 September 18, and the lower two spectra are from WIYN Hydra on 2001 August 24 and September 9. The broad interstellar band feature near $4428 \AA$ is absent in the high-resolution Keck spectrum because it has been fitted and removed during continuum normalization. The ratio of $\mathrm{He}_{\mathrm{I}} \lambda 4471$ to $\mathrm{Mg}$ II $\lambda 4481$ changes from over $2: 1$ to near $1: 1$. The strength of the Si II $\lambda \lambda 4128,4130$ doublet becomes stronger than the adjacent He I lines. N III $\lambda 4097$ becomes noticeably weaker than $\mathrm{H} \delta \lambda 4101$, and $\mathrm{Fe}$ II $\lambda 4232$ becomes much stronger. In addition, the Balmer line strength also increases. Emission in the Balmer and higher ionization lines is also less dominant in the 2001 August and September spectra, which are best described by a classification of B6 and B8. It is more dominant in the 2000 September spectrum, which is best described by a classification of B3. A sign of late B classification is the appearance of the $\mathrm{Mg}$ I doublet at 5173 and $5184 \AA$; however, the August and September WIYN spectra did not cover 
TABLE 2

Early-Type Stars in the Direction of Cyg OB2

\begin{tabular}{|c|c|c|c|c|c|c|c|c|c|c|c|c|c|c|c|}
\hline $\begin{array}{l}\text { Star } \\
\text { (MT) } \\
(1)\end{array}$ & $\begin{array}{c}\text { Lit S.C. } \\
\text { (2) }\end{array}$ & $\begin{array}{c}\text { Our S.C. } \\
\text { (3) }\end{array}$ & $\begin{array}{l}\text { R.A. } \\
\text { (J2000.0) } \\
(4)\end{array}$ & $\begin{array}{c}\text { Decl. } \\
(\mathrm{J} 2000.0) \\
(5)\end{array}$ & $\begin{array}{l}A_{V} \\
\text { (6) }\end{array}$ & $\begin{array}{c}V \\
(7)\end{array}$ & $\begin{array}{l}M_{V} \\
(8)\end{array}$ & $\begin{array}{c}(B-V) \\
\quad(9)\end{array}$ & $\begin{array}{c}(B-V)_{0} \\
\quad(10)\end{array}$ & $\begin{array}{l}M_{\mathrm{PD}} \\
\left(M_{\odot}\right) \\
(11)\end{array}$ & $\begin{array}{c}M_{0} \\
\left(M_{\odot}\right) \\
(12)\end{array}$ & $\begin{array}{l}\text { DM } \\
\text { (13) }\end{array}$ & $\begin{array}{l}\text { Notes } \\
(14)\end{array}$ & $\begin{array}{l}\text { Ph. Activ. } \\
\text { (15) }\end{array}$ & $\begin{array}{l}\text { Ref. } \\
\text { (16) }\end{array}$ \\
\hline 5 & $\mathrm{O} 6 \mathrm{~V}$ & $\mathrm{O} 6 \mathrm{~V}$ & 203039.87 & +413650.9 & 5.8 & 12.93 & -4.92 & 1.64 & -0.30 & 31.7 & 31.7 & 12.0 & & & \\
\hline 20 & & B $0 \mathrm{~V}$ & 203051.12 & +412021.6 & 7.3 & 14.48 & -3.80 & 2.18 & -0.26 & 17.5 & 17.5 & 10.9 & $\mathrm{n}$ & & \\
\hline $21 \ldots$ & B2: & B2 II & 203050.75 & +413506.0 & 4.4 & 13.74 & -4.80 & 1.30 & -0.18 & 18.9 & 20.0 & 14.1 & & & \\
\hline 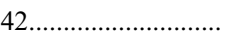 & & B2 V & 203059.43 & +413559.6 & 4.4 & 13.73 & -2.50 & 1.27 & -0.21 & 10.0 & 10.0 & 11.7 & $\mathrm{n}$ & & \\
\hline 59 & $08.5 \mathrm{~V}$ & $\mathrm{O} 8 \mathrm{~V}$ & 203110.57 & +413153.0 & 5.3 & 11.18 & -4.34 & 1.47 & -0.28 & 22.0 & 22.0 & 10.2 & & & \\
\hline 70 & O9 V & $\mathrm{O} 9 \mathrm{~V}$ & 203118.31 & +412121.7 & 7.1 & 12.99 & -4.05 & 2.10 & -0.28 & 18.0 & 18.0 & 10.0 & & & \\
\hline $83 \ldots$ & B1 I & B1 I & 203122.03 & +413128.0 & 4.1 & 10.64 & -6.50 & 1.18 & -0.19 & 24.0 & 26.0 & 13.0 & & Susp & 1 \\
\hline $97 \ldots$ & $\ldots$ & B2 V & 203130.49 & +413715.6 & 4.3 & 14.56 & -2.50 & 1.22 & -0.21 & 10.0 & 10.0 & 12.7 & $\mathrm{n}$ & & \\
\hline $103 \ldots \ldots \ldots \ldots \ldots \ldots \ldots \ldots \ldots . .$. & $\ldots$ & B1 V & 203133.38 & +412249.0 & 6.7 & 13.81 & -3.20 & 2.00 & -0.23 & 13.8 & 13.8 & 10.3 & $\mathrm{n}$ & & \\
\hline $106 \ldots$ & $\ldots$ & B3 V & 203133.59 & +413604.3 & 4.4 & 14.60 & -1.90 & 1.29 & -0.18 & 7.6 & 7.6 & 12.0 & $\mathrm{n}$ & & \\
\hline $108 \ldots \ldots \ldots \ldots \ldots$ & $\ldots$ & B3 IV & 203134.12 & +413108.0 & 4.3 & 14.88 & -2.50 & 1.26 & -0.16 & 7.6 & 7.6 & 13.1 & $\mathrm{n}$ & & \\
\hline $129 \ldots \ldots \ldots \ldots \ldots \ldots$ & $\ldots$ & B3 V & 203141.60 & +412820.9 & 4.6 & 14.40 & -1.90 & 1.34 & -0.18 & 7.6 & 7.6 & 11.7 & $\mathrm{n}$ & & \\
\hline $138 \ldots \ldots \ldots$ & O8.5 I & O8 I & 203145.39 & +411826.8 & 6.9 & 12.26 & -6.30 & 1.99 & -0.30 & 36.8 & 39.8 & 11.6 & & & \\
\hline $145 \ldots$ & $09.5 \mathrm{~V}$ & O9 III & 203149.65 & +412826.8 & 4.1 & 11.52 & -5.25 & 1.11 & -0.26 & 23.1 & 24.6 & 12.6 & & & \\
\hline $164 \ldots$ & $\ldots$ & B3 V & 203155.28 & +413527.8 & 4.3 & 15.07 & -1.90 & 1.25 & -0.18 & 7.6 & 7.6 & 12.6 & $\mathrm{n}$ & & \\
\hline $169 \ldots \ldots \ldots \ldots \ldots \ldots \ldots . .$. & $\mathrm{B} 1.5 \mathrm{~V}$ & $\mathrm{~B} 2 \mathrm{~V}$ & 203156.27 & +413305.3 & 4.3 & 13.90 & -2.50 & 1.21 & -0.21 & 10.0 & 10.0 & 12.1 & & & \\
\hline 170 & $\ldots$ & B5: V & 203156.23 & +413512.3 & 3.6 & 15.21 & -1.35 & 1.05 & -0.15 & 5.9 & 5.9 & 11.6 & $\mathrm{n}$ & & \\
\hline $174 \ldots \ldots \ldots$ & $\mathrm{B} 2 \mathrm{~V}$ & B2 IV & 203156.90 & +413148.0 & 4.3 & 12.55 & -3.10 & 1.21 & -0.21 & 10.0 & 10.0 & 11.3 & & & \\
\hline $179 \ldots \ldots$ & $\ldots$ & B3 V & 203159.82 & +413714.3 & 4.9 & 14.00 & -1.90 & 1.46 & -0.18 & 7.6 & 7.6 & 10.9 & $\mathrm{n}$ & & \\
\hline $186 \ldots \ldots \ldots \ldots$ & $\ldots$ & $\mathrm{B} 2 \mathrm{Ve}$ & 203203.01 & +413230.7 & 4.2 & 14.14 & -2.50 & 1.20 & -0.21 & 10.0 & 10.0 & 12.4 & $\mathrm{n}$ & & \\
\hline 187...…………...... & B1 V & B1 V & 203203.74 & +412510.9 & 5.3 & 13.24 & -3.20 & 1.52 & -0.23 & 13.8 & 13.8 & 11.1 & & & \\
\hline $189 \ldots \ldots \ldots$ & $\ldots$ & B6: V & 203204.57 & +412748.6 & 3.9 & 14.77 & -1.15 & 1.17 & -0.14 & 5.4 & 5.4 & 10.8 & $\mathrm{n}$ & & \\
\hline $191 \ldots \ldots$ & $\ldots$ & B3 IV & 203204.74 & +412844.5 & 3.6 & 13.81 & -2.50 & 1.04 & -0.16 & 7.6 & 7.6 & 12.7 & $\mathrm{n}$ & & \\
\hline $196 \ldots$ & $\ldots$ & B6 V & 203205.59 & +412749.6 & 4.4 & 14.81 & -1.15 & 1.31 & -0.14 & 5.4 & 5.4 & 10.4 & $\mathrm{n}$ & & \\
\hline 200 & $\ldots$ & B3 V & 203206.85 & +411756.8 & 6.5 & 13.94 & -1.90 & 1.98 & -0.18 & 7.6 & 7.6 & 9.4 & $\mathrm{n}$ & & \\
\hline $202 \ldots \ldots \ldots \ldots \ldots \ldots \ldots$ & $\ldots$ & B2 V & 203207.95 & +412200.3 & 4.7 & 14.40 & -2.50 & 1.35 & -0.21 & 10.0 & 10.0 & 12.2 & $\mathrm{n}$ & & \\
\hline $213 \ldots \ldots \ldots \ldots \ldots \ldots \ldots \ldots \ldots$ & B $0 \mathrm{~V}$ & B $0 \mathrm{~V}$ & 203213.07 & +412724.9 & 4.2 & 11.95 & -3.80 & 1.13 & -0.26 & 17.5 & 17.5 & 11.5 & & & \\
\hline $215 \ldots \ldots \ldots \ldots \ldots \ldots \ldots \ldots \ldots . .$. & B1 V & B2 V & $2032 \quad 13.48$ & +412731.0 & 3.5 & 12.97 & -2.50 & 0.96 & -0.21 & 10.0 & 10.0 & 11.9 & & & \\
\hline 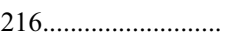 & $\ldots$ & $\mathrm{B} 1.5 \mathrm{~V}$ & 203213.75 & +412742.0 & 4.2 & 13.02 & -2.80 & 1.18 & -0.22 & 11.9 & 11.9 & 11.6 & $\mathrm{n}$ & & \\
\hline $217 \ldots \ldots$ & O7 IIIf & O7 IIIf & $2032 \quad 13.77$ & +412712.7 & 4.4 & 10.23 & -5.54 & 1.19 & -0.29 & 31.2 & 33.0 & 11.3 & & & \\
\hline $220 \ldots \ldots \ldots \ldots \ldots \ldots$ & $\ldots$ & B1 V & 203214.56 & +412233.7 & 5.3 & 14.34 & -3.20 & 1.52 & -0.23 & 13.8 & 13.8 & 12.2 & $\mathrm{n}$ & & \\
\hline 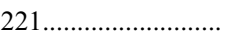 & $\ldots$ & B2 V & 203214.63 & +412740.3 & 4.5 & 13.62 & -2.50 & 1.30 & -0.21 & 10.0 & 10.0 & 11.5 & $\mathrm{n}$ & & \\
\hline 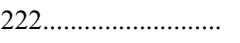 & $\ldots$ & B3 V & 203215.03 & +411930.8 & 5.0 & 14.80 & -1.90 & 1.47 & -0.18 & 7.6 & 7.6 & 11.7 & $\mathrm{n}$ & & \\
\hline 227 & $\mathrm{O} 9 \mathrm{~V}$ & $\mathrm{O} 9 \mathrm{~V}$ & $2032 \quad 16.53$ & +412536.4 & 4.6 & 11.47 & -4.05 & 1.24 & -0.28 & 18.0 & 18.0 & 10.9 & & & \\
\hline $234 \ldots \ldots \ldots \ldots$ & $\ldots$ & $\mathrm{B} 2 \mathrm{~V}$ & 203219.66 & +412039.7 & 4.5 & 13.25 & -2.50 & 1.28 & -0.21 & 10.0 & 10.0 & 11.2 & $\mathrm{n}$ & & \\
\hline $238 \ldots \ldots$ & $\ldots$ & B1 V & $2032 \quad 21.35$ & +411835.5 & 6.1 & 14.91 & -3.20 & 1.80 & -0.23 & 13.8 & 13.8 & 12.0 & $\mathrm{n}$ & & \\
\hline 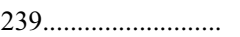 & $\ldots$ & B4 V & 203221.76 & +413424.6 & 3.9 & 14.33 & -1.65 & 1.14 & -0.16 & 6.4 & 6.4 & 10.4 & $\mathrm{n}$ & & \\
\hline $241 \ldots \ldots \ldots \ldots$ & $\ldots$ & B2 V & 203222.15 & +412741.7 & 4.0 & 13.41 & -2.50 & 1.13 & -0.21 & 10.0 & 10.0 & 11.8 & $\mathrm{n}$ & & \\
\hline 248 & $\ldots$ & $\mathrm{B} 2 \mathrm{~V}$ & 203225.50 & +412451.8 & 4.6 & 13.36 & -2.50 & 1.31 & -0.21 & 10.0 & 10.0 & 11.2 & & & \\
\hline $250 \ldots \ldots \ldots \ldots \ldots \ldots \ldots$ & $\mathrm{B} 1 \mathrm{~V}$ & B2 III & 203226.10 & +412939.0 & 3.8 & 12.88 & -3.70 & 1.06 & -0.19 & 14.8 & 15.0 & 12.8 & & & \\
\hline $252 \ldots \ldots \ldots \ldots \ldots \ldots \ldots \ldots . .$. & $\ldots$ & B1.5 III & 203226.50 & +411913.7 & 4.9 & 14.15 & -3.90 & 1.42 & -0.20 & 16.1 & 16.3 & 13.1 & $\mathrm{n}$ & & \\
\hline $255 \ldots \ldots \ldots \ldots \ldots \ldots \ldots \ldots . .$. & $\ldots$ & B2 III & 203227.26 & +412156.2 & 4.8 & 14.71 & -3.70 & 1.42 & -0.19 & 14.8 & 15.0 & 13.5 & $\mathrm{n}$ & & \\
\hline 258 & $\mathrm{O} 8 \mathrm{~V}$ & $\mathrm{O} 8 \mathrm{~V}$ & 203227.67 & +412621.7 & 4.5 & 11.10 & -4.34 & 1.20 & -0.28 & 22.0 & 22.0 & 10.9 & & & \\
\hline 259 & B1 V & B0 Ib & $2032 \quad 27.76$ & +412851.9 & 3.7 & 11.42 & -6.00 & 1.00 & -0.22 & 25.0 & 27.0 & 13.7 & & & \\
\hline $264 \ldots \ldots \ldots \ldots \ldots \ldots \ldots \ldots$ & $\ldots$ & B2 III & 203230.72 & +410704.1 & 3.5 & 12.63 & -3.70 & 0.99 & -0.19 & 14.8 & 15.0 & 12.7 & $\mathrm{n}$ & & \\
\hline
\end{tabular}


TABLE 2-Continued

\begin{tabular}{|c|c|c|c|c|c|c|c|c|c|c|c|c|c|c|c|}
\hline $\begin{array}{l}\text { Star } \\
\text { (MT) } \\
(1)\end{array}$ & $\begin{array}{l}\text { Lit S.C. } \\
\text { (2) }\end{array}$ & $\begin{array}{l}\text { Our S.C. } \\
\text { (3) }\end{array}$ & $\begin{array}{c}\text { R.A. } \\
\text { (J2000.0) } \\
\text { (4) }\end{array}$ & $\begin{array}{c}\text { Decl. } \\
(\mathrm{J} 2000.0) \\
(5)\end{array}$ & $\begin{array}{l}A_{V} \\
(6)\end{array}$ & $\begin{array}{c}V \\
(7)\end{array}$ & $\begin{array}{l}M_{V} \\
(8)\end{array}$ & $\begin{array}{c}(B-V) \\
(9)\end{array}$ & $\begin{array}{c}(B-V)_{0} \\
\quad(10)\end{array}$ & $\begin{array}{l}M_{\mathrm{PD}} \\
\left(M_{\odot}\right) \\
(11)\end{array}$ & $\begin{array}{c}M_{0} \\
\left(M_{\odot}\right) \\
(12)\end{array}$ & $\begin{array}{l}\text { DM } \\
\text { (13) }\end{array}$ & $\begin{array}{c}\text { Notes } \\
(14)\end{array}$ & $\begin{array}{l}\text { Ph. Activ. } \\
\text { (15) }\end{array}$ & $\begin{array}{l}\text { Ref. } \\
\text { (16) }\end{array}$ \\
\hline $268 \ldots \ldots \ldots \ldots \ldots \ldots \ldots \ldots .$. & $\ldots$ & B $2.5 \mathrm{~V}$ & 203231.42 & +413051.4 & 4.9 & 14.38 & -2.20 & 1.43 & -0.19 & 8.8 & 8.8 & 11.7 & $\mathrm{n}$ & & \\
\hline $271 \ldots \ldots \ldots \ldots \ldots \ldots \ldots \ldots$ & $\ldots$ & B4 V & 203232.34 & +412257.6 & 5.0 & 14.57 & -1.65 & 1.51 & -0.16 & 6.4 & 6.4 & 9.5 & $\mathrm{n}$ & & \\
\hline $273 \ldots \ldots \ldots \ldots \ldots \ldots \ldots \ldots$ & $\ldots$ & B5: V & 203232.54 & +412646.7 & 5.2 & 14.91 & -1.35 & 1.57 & -0.15 & 5.9 & 5.9 & 9.7 & $\mathrm{n}$ & & \\
\hline $275 \ldots \ldots \ldots \ldots \ldots \ldots \ldots \ldots$ & $\ldots$ & $\mathrm{B} 2 \mathrm{~V}$ & 203232.68 & +412704.4 & 3.9 & 13.47 & -2.50 & 1.10 & -0.21 & 10.0 & 10.0 & 12.0 & $\mathrm{n}$ & & \\
\hline 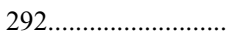 & $\mathrm{B} 1 \mathrm{~V}$ & $\mathrm{~B} 2 \mathrm{~V}$ & 203237.03 & +412305.1 & 5.2 & 13.08 & -2.50 & 1.51 & -0.21 & 10.0 & 10.0 & 10.4 & & & \\
\hline $295 \ldots \ldots \ldots \ldots \ldots \ldots \ldots \ldots . .$. & $\ldots$ & $\mathrm{B} 2 \mathrm{~V}$ & 203237.78 & +412615.3 & 4.3 & 13.71 & -2.50 & 1.21 & -0.21 & 10.0 & 10.0 & 11.9 & $\mathrm{n}$ & & \\
\hline $298 \ldots \ldots \ldots \ldots \ldots \ldots \ldots \ldots . .$. & $\ldots$ & B3 V & 203238.34 & +412856.6 & 4.3 & 14.43 & -1.90 & 1.25 & -0.18 & 7.6 & 7.6 & 12.0 & $\mathrm{n}$ & & \\
\hline 299 & $\mathrm{O} 7 \mathrm{~V}$ & $\mathrm{O} 7 \mathrm{~V}$ & 203238.58 & +412513.6 & 4.4 & 10.84 & -4.63 & 1.19 & -0.29 & 26.5 & 26.5 & 11.0 & & & \\
\hline $300 \ldots \ldots \ldots \ldots \ldots \ldots \ldots$ & B1: & B1 V & 203238.87 & +412520.8 & 4.3 & 13.05 & -3.20 & 1.21 & -0.23 & 13.8 & 13.8 & 11.9 & & & \\
\hline 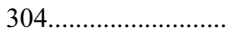 & B5 Iae & B3 Iae & 203240.88 & +411429.3 & 10. & 11.46 & -6.30 & 3.35 & -0.13 & 22.0 & 23.5 & 7.4 & & Susp & 1 \\
\hline $311 \ldots \ldots$ & $\ldots$ & B2 V & 203242.90 & +412016.4 & 4.8 & 13.87 & -2.50 & 1.39 & -0.21 & 10.0 & 10.0 & 11.5 & $\mathrm{n}$ & & \\
\hline 317................................ & $\mathrm{O} 8 \mathrm{~V}$ & $\mathrm{O} 8 \mathrm{~V}$ & 203245.45 & +412537.3 & 4.6 & 10.68 & -4.34 & 1.25 & -0.28 & 22.0 & 22.0 & 10.4 & & & \\
\hline $322 \ldots \ldots \ldots \ldots \ldots \ldots \ldots \ldots . .$. & $\ldots$ & $\mathrm{B} 2.5 \mathrm{~V}$ & 203246.45 & +412422.4 & 4.6 & 14.91 & -2.20 & 1.33 & -0.19 & 8.8 & 8.8 & 12.5 & $\mathrm{n}$ & & \\
\hline 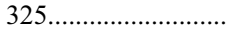 & $\ldots$ & B1.5 III & 203246.74 & +412615.9 & 4.7 & 14.30 & -3.90 & 1.37 & -0.20 & 16.1 & 16.3 & 13.4 & $\mathrm{n}$ & & \\
\hline $336 \ldots \ldots \ldots \ldots \ldots \ldots$ & $\ldots$ & B3 III & 203249.67 & +412536.4 & 4.0 & 14.13 & -3.00 & 1.17 & -0.16 & 12.2 & 12.3 & 13.1 & $\mathrm{n}$ & & \\
\hline $339 \ldots \ldots \ldots \ldots \ldots \ldots \ldots \ldots \ldots$ & $08.5 \mathrm{~V}$ & $\mathrm{O} 8 \mathrm{~V}$ & 203250.03 & +412344.6 & 4.9 & 11.60 & -4.34 & 1.35 & -0.28 & 22.0 & 22.0 & 11.0 & & & \\
\hline 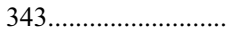 & $\ldots$ & B1 V & 203250.69 & +411502.2 & 6.6 & 14.44 & -3.20 & 1.98 & -0.23 & 13.8 & 13.8 & 11.0 & $\mathrm{n}$ & & \\
\hline 358 & $\mathrm{~B}$ & B3 V & 203254.35 & +411522.1 & 7.0 & 14.81 & -1.90 & 2.16 & -0.18 & 7.6 & 7.6 & 9.7 & & & \\
\hline 365 & $\ldots$ & $\mathrm{B} 1 \mathrm{~V}$ & 203256.66 & +412341.0 & 4.5 & 13.81 & -3.20 & 1.28 & -0.23 & 13.8 & 13.8 & 12.4 & $\mathrm{n}$ & & \\
\hline 372 …… & $\ldots$ & B0 V & 203258.79 & +410429.9 & 7.3 & 14.97 & -3.80 & 2.17 & -0.26 & 17.5 & 17.5 & 11.4 & $\mathrm{n}$ & & \\
\hline 376 ................................ & $\mathrm{O} 8 \mathrm{~V}$ & $\mathrm{O} 8 \mathrm{~V}$ & 203259.17 & +412425.7 & 4.9 & 11.91 & -4.34 & 1.35 & -0.28 & 22.0 & 22.0 & 11.3 & & & \\
\hline 378 & B0 V & B0 V & 203259.61 & +411514.6 & 7.1 & 13.49 & -3.80 & 2.10 & -0.26 & 17.5 & 17.5 & 10.2 & & & \\
\hline 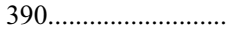 & $\mathrm{O} 8 \mathrm{~V}$ & $\mathrm{O} 8 \mathrm{~V}$ & 203302.94 & +411743.3 & 6.8 & 12.95 & -4.34 & 1.98 & -0.28 & 22.0 & 22.0 & 10.4 & & Irr & 2 \\
\hline $395 \ldots \ldots \ldots \ldots \ldots \ldots$ & $\mathrm{B} 1.5 \mathrm{~V}$ & $\mathrm{~B} 1 \mathrm{~V}$ & 203304.42 & +411708.9 & 6.0 & 14.14 & -3.20 & 1.75 & -0.23 & 13.8 & 13.8 & 11.4 & $\mathrm{n}$ & & \\
\hline $400 \ldots \ldots \ldots \ldots \ldots \ldots \ldots \ldots$ & B & $\mathrm{B} 1 \mathrm{~V}$ & 203305.22 & +41 1751.6 & 5.6 & 14.15 & -3.20 & 1.62 & -0.23 & 13.8 & 13.8 & 11.8 & & & \\
\hline 403 & $\mathrm{~B} 2 \mathrm{~V}$ & B1 V & 203305.55 & +414337.2 & 5.2 & 12.94 & -3.20 & 1.49 & -0.23 & 13.8 & 13.8 & 10.9 & & & \\
\hline 409 & & B $0.5 \mathrm{~V}$ & 203306.62 & +412113.3 & 6.0 & 14.21 & -3.80 & 1.76 & -0.24 & 15.6 & 15.6 & 12.0 & $\mathrm{n}$ & & \\
\hline 417............................ & O4 III & O4 III & 203308.78 & +411318.1 & 7.1 & 11.55 & -5.98 & 2.04 & -0.31 & 48.8 & 51.0 & 10.4 & & & \\
\hline 420 & $\ldots$ & O9 V & 203309.41 & +411258.2 & 6.8 & 12.84 & -4.05 & 1.97 & -0.28 & 18.0 & 18.0 & 10.1 & $\mathrm{n}, \mathrm{b}$ & & \\
\hline $421 \ldots \ldots \ldots \ldots \ldots \ldots \ldots$ & O9 V & $\mathrm{O} 9 \mathrm{~V}$ & 203309.58 & +411300.6 & 6.7 & 12.86 & -4.05 & 1.96 & -0.28 & 18.0 & 18.0 & 10.1 & $\mathrm{~b}$ & EA, $P=4.161$ days & 2 \\
\hline $425 \ldots \ldots \ldots \ldots \ldots \ldots \ldots$ & B0 V & B0 V & 203310.10 & +411310.1 & 6.6 & 13.62 & -3.80 & 1.94 & -0.26 & 17.5 & 17.5 & 10.8 & & & \\
\hline 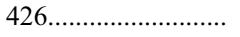 & B0 V & B0 V & $2033 \quad 10.34$ & +41 1306.4 & 6.6 & 14.05 & -3.80 & 1.95 & -0.26 & 17.5 & 17.5 & 11.2 & & & \\
\hline 427......................... & $\ldots$ & B4 II-B4 III & $2033 \quad 10.27$ & +412344.9 & 4.6 & 14.97 & -4.60 & 1.39 & -0.13 & 15.3 & 15.5 & 10.4 & $\mathrm{n}$ & & \\
\hline 428.......................... & $\ldots$ & B1 V & 203310.46 & +412057.6 & 6.0 & 14.06 & -3.20 & 1.77 & -0.23 & 13.8 & 13.8 & 11.2 & $\mathrm{n}$ & & \\
\hline $429 \ldots \ldots \ldots \ldots \ldots \ldots \ldots \ldots$ & B0 V & B0 V & 203310.50 & +412222.8 & 5.5 & 12.98 & -3.80 & 1.56 & -0.26 & 17.5 & 17.5 & 11.3 & & EA; $P=2.9788$ days: & 2 \\
\hline 431............................. & O5 If & O5 If & 203310.74 & +411508.0 & 6.4 & 10.96 & -6.33 & 1.81 & -0.32 & 50.9 & 53.0 & 10.9 & & Unk; $P=1.22 / 5.6$ days & 2 \\
\hline $435 \ldots \ldots \ldots \ldots \ldots \ldots \ldots \ldots$ & $\ldots$ & B0 V & 203311.02 & +41 1031.9 & 7.4 & 14.78 & -3.80 & 2.19 & -0.26 & 17.5 & 17.5 & 11.2 & $\mathrm{n}$ & & \\
\hline $441 \ldots \ldots \ldots \ldots \ldots \ldots \ldots \ldots \ldots$ & $\ldots$ & B2 III: & 203311.39 & +411758.9 & 5.1 & 14.38 & -3.70 & 1.52 & -0.19 & 14.8 & 15.0 & 12.9 & $\mathrm{n}$ & & \\
\hline 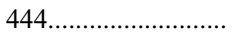 & $\ldots$ & B5 V: & 203311.81 & +412405.8 & 4.9 & 14.12 & -1.35 & 1.48 & -0.15 & 5.9 & 5.9 & 9.3 & $\mathrm{n}$ & & \\
\hline 448................................ & O6 V & $\mathrm{O} 6 \mathrm{~V}$ & $2033 \quad 13.25$ & +411328.6 & 7.4 & 13.61 & -4.92 & 2.15 & -0.30 & 31.7 & 31.7 & 11.1 & & Unk; $P=3.16$ days: & 2 \\
\hline 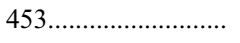 & $\ldots$ & B5: V & 203313.37 & +412639.7 & 3.9 & 14.45 & -1.35 & 1.14 & -0.15 & 5.9 & 5.9 & 10.5 & $\mathrm{n}$ & & \\
\hline 455................................. & O8 V & $08 \mathrm{~V}$ & 203313.67 & +411305.7 & 6.3 & 12.92 & -4.34 & 1.81 & -0.28 & 22.0 & 22.0 & 10.9 & & & \\
\hline 457........................... & $\mathrm{O} 3$ If & $\mathrm{O} 3$ If & 203314.16 & +412021.5 & 5.4 & 10.55 & -6.35 & 1.45 & -0.34 & 80.0 & 100 & 11.5 & & Irr & 2 \\
\hline 459......................... & $\ldots$ & B5: & 203314.34 & +41 1933.0 & 5.8 & 14.67 & -1.35 & 1.79 & -0.15 & 5.9 & 5.9 & 8.8 & $\mathrm{n}$ & & \\
\hline $462 \ldots$ & O6.5 III & O7 III-II & 203314.84 & +41 1841.4 & 5.2 & 10.33 & -5.54 & 1.44 & -0.29 & 31.2 & 33.0 & 10.6 & & Cst & 2 \\
\hline $465 \ldots \ldots \ldots \ldots \ldots \ldots \ldots \ldots$ & O5.5 I & O5.5 I & 203315.18 & +411850.1 & 4.9 & 9.06 & -6.33 & 1.30 & -0.32 & 48.3 & 50.3 & 10.5 & & & \\
\hline 467...…………....... & B1 V & B1 V & $2033 \quad 15.37$ & +412956.6 & 5.5 & 13.43 & -3.20 & 1.59 & -0.23 & 13.8 & 13.8 & 11.1 & & & \\
\hline
\end{tabular}


TABLE 2-Continued

\begin{tabular}{|c|c|c|c|c|c|c|c|c|c|c|c|c|c|c|c|}
\hline $\begin{array}{l}\text { Star } \\
\text { (MT) } \\
(1)\end{array}$ & $\begin{array}{l}\text { Lit S.C. } \\
\text { (2) }\end{array}$ & $\begin{array}{l}\text { Our S.C. } \\
\text { (3) }\end{array}$ & $\begin{array}{c}\text { R.A. } \\
(\text { J2000.0) } \\
(4)\end{array}$ & $\begin{array}{c}\text { Decl. } \\
(\mathrm{J} 2000.0) \\
(5)\end{array}$ & $\begin{array}{l}A_{V} \\
(6)\end{array}$ & $\begin{array}{c}V \\
(7)\end{array}$ & $\begin{array}{c}M_{V} \\
(8)\end{array}$ & $\begin{array}{c}(B-V) \\
(9)\end{array}$ & $\begin{array}{c}(B-V)_{0} \\
\quad(10)\end{array}$ & $\begin{array}{l}M_{\mathrm{PD}} \\
\left(M_{\odot}\right) \\
(11)\end{array}$ & $\begin{array}{l}M_{0} \\
\left(M_{\odot}\right) \\
(12)\end{array}$ & $\begin{array}{l}\mathrm{DM} \\
(13)\end{array}$ & $\begin{array}{l}\text { Notes } \\
(14)\end{array}$ & $\begin{array}{l}\text { Ph. Activ. } \\
\text { (15) }\end{array}$ & $\begin{array}{l}\text { Ref. } \\
\text { (16) }\end{array}$ \\
\hline $469 \ldots \ldots \ldots \ldots \ldots \ldots \ldots$ & $\ldots$ & B1 III & 203315.51 & +412732.9 & 4.9 & 13.65 & -4.30 & 1.41 & -0.21 & 17.5 & 18.3 & 13.0 & $\mathrm{n}$ & & \\
\hline 470 & O9 V & O9 V & $2033 \quad 15.74$ & +412017.2 & 5.2 & 12.50 & -4.05 & 1.46 & -0.28 & 18.0 & 18.0 & 11.3 & & Cst & 2 \\
\hline 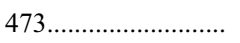 & $08.5 \mathrm{~V}$ & $08.5 \mathrm{~V}$ & 203316.36 & +411901.9 & 5.2 & 12.02 & -4.19 & 1.45 & -0.28 & 19.8 & 19.8 & 11.0 & & Cst & 2 \\
\hline $477 \ldots \ldots$ & $\ldots$ & B0: V & 203317.40 & +411238.7 & 6.5 & 14.43 & -3.80 & 1.91 & -0.26 & 17.5 & 17.5 & 11.7 & $\mathrm{n}$ & & \\
\hline $480 \ldots \ldots \ldots \ldots \ldots \ldots \ldots \ldots$ & $\mathrm{O} 7 \mathrm{~V}$ & $\mathrm{O} 7 \mathrm{~V}$ & 203317.49 & +411709.2 & 5.6 & 11.88 & -4.63 & 1.59 & -0.29 & 26.5 & 26.5 & 10.8 & & Cst & 2 \\
\hline $483 \ldots \ldots \ldots \ldots \ldots \ldots \ldots . .$. & O5 I & O5 III & 203318.02 & +411831.0 & 4.6 & 10.19 & -5.84 & 1.24 & -0.30 & 41.5 & 43.0 & 11.4 & & Cst & 2 \\
\hline 485 & $\mathrm{O} 8 \mathrm{~V}$ & $\mathrm{O} 8 \mathrm{~V}$ & 203318.08 & +412136.6 & 5.4 & 12.06 & -4.34 & 1.51 & -0.28 & 22.0 & 22.0 & 11.0 & & Cst & 2 \\
\hline 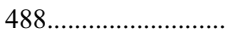 & $\mathrm{Be}$ & $\mathrm{B} 1 \mathrm{Ve}-\mathrm{B} 3 \mathrm{Ve}$ & 203318.55 & +411535.4 & 7.7 & 14.88 & -3.20 & 2.36 & -0.21 & 10.0 & 10.0 & 10.3 & & $\mathrm{BE}$ & 2 \\
\hline $490 \ldots \ldots \ldots \ldots \ldots \ldots \ldots \ldots \ldots$ & $\ldots$ & B0: & 203318.56 & +412449.3 & 5.4 & 14.76 & -3.80 & 1.53 & -0.26 & 17.5 & 17.5 & 13.1 & $\mathrm{n}$ & & \\
\hline $492 \ldots \ldots$ & $\ldots$ & B1 V & 203319.16 & +411744.9 & 5.7 & 14.85 & -3.20 & 1.68 & -0.23 & 13.8 & 13.8 & 12.3 & $\mathrm{n}$ & & \\
\hline 493 & $\ldots$ & B5 IV & 203319.26 & +412444.8 & 5.3 & 14.99 & -1.35 & 1.61 & -0.15 & 5.9 & 5.9 & 9.7 & $\mathrm{n}$ & & \\
\hline $507 \ldots \ldots$ & $\mathrm{O} 9 \mathrm{~V}$ & O9 V & 203321.04 & +411740.1 & 5.5 & 12.70 & -4.05 & 1.54 & -0.28 & 18.0 & 18.0 & 11.2 & & Cst & 2 \\
\hline $509 \ldots \ldots \ldots \ldots \ldots \ldots \ldots$ & $\ldots$ & B0 III-B0 IV & 203321.14 & +413552.0 & 6.1 & 14.72 & -5.00 & 1.79 & -0.23 & 20.0 & 20.8 & 13.6 & $\mathrm{n}$ & & \\
\hline 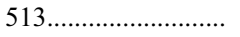 & $\ldots$ & B2 V & 203322.49 & +412216.9 & 4.9 & 14.26 & -2.50 & 1.42 & -0.21 & 10.0 & 10.0 & 11.8 & $\mathrm{n}$ & & \\
\hline $515 \ldots \ldots \ldots \ldots \ldots \ldots \ldots \ldots \ldots \ldots \ldots \ldots \ldots \ldots \ldots . .$. & $\mathrm{B} 1 \mathrm{~V}$ & $\mathrm{~B} 1 \mathrm{~V}$ & 203323.24 & +411341.9 & 6.8 & 14.66 & -3.20 & 2.03 & -0.23 & 13.8 & 13.8 & 11.0 & & & \\
\hline $516 \ldots \ldots \ldots \ldots \ldots \ldots \ldots$ & $\mathrm{O} 5.5 \mathrm{~V}$ & $05.5 \mathrm{~V}$ & 203323.46 & +410912.9 & 7.5 & 11.84 & -5.07 & 2.20 & -0.30 & 34.2 & 34.2 & 9.5 & & & \\
\hline $517 \ldots \ldots \ldots \ldots \ldots \ldots \ldots$ & $\ldots$ & B1 V & 203323.37 & +412017.2 & 5.2 & 13.74 & -3.20 & 1.50 & -0.23 & 13.8 & 13.8 & 11.7 & $\mathrm{n}$ & & \\
\hline $522 \ldots \ldots \ldots \ldots \ldots \ldots \ldots \ldots \ldots$ & $\ldots$ & B2 Ve: & 203324.78 & +412204.5 & 4.8 & 14.06 & -2.50 & 1.38 & -0.21 & 10.0 & 10.0 & 11.7 & $\mathrm{n}$ & BCEP $: ; ~ P=0.21210$ days? & 2 \\
\hline $531 \ldots \ldots \ldots \ldots \ldots \ldots \ldots \ldots$ & $08.5 \mathrm{~V}$ & $08.5 \mathrm{~V}$ & 203329.42 & +412154.1 & 5.6 & 11.58 & -4.19 & 1.57 & -0.28 & 19.8 & 19.8 & 10.2 & & & \\
\hline $534 \ldots \ldots \ldots \ldots \ldots \ldots \ldots$ & $07.5 \mathrm{~V}$ & $08.5 \mathrm{~V}$ & 203326.77 & +411059.5 & 6.5 & 13.00 & -4.19 & 1.87 & -0.28 & 19.8 & 19.8 & 10.7 & & & \\
\hline 539 & $\ldots$ & B5: Ve & 203327.21 & +413557.8 & 7.3 & 14.61 & -1.35 & 2.29 & -0.15 & 5.9 & 5.9 & 7.3 & $\mathrm{n}$ & & \\
\hline $554 \ldots \ldots \ldots \ldots \ldots \ldots \ldots \ldots \ldots$ & $\ldots$ & $\mathrm{B} 4 \mathrm{Ve}$ & 203330.55 & +412017.3 & 4.7 & 14.41 & -1.65 & 1.41 & -0.16 & 6.4 & 6.4 & 9.7 & $\mathrm{n}$ & EA & 2 \\
\hline $555 \ldots \ldots \ldots \ldots \ldots \ldots \ldots$ & $\mathrm{O} 8 \mathrm{~V}$ & & 203330.43 & +413557.5 & 6.6 & 12.51 & -4.34 & 1.90 & -0.28 & 22.0 & 22.0 & 10.2 & & & \\
\hline $556 \ldots \ldots \ldots \ldots \ldots \ldots \ldots \ldots$ & B1 I & B1 I & 203330.81 & +411522.7 & 5.9 & 11.01 & -6.50 & 1.77 & -0.19 & 24.0 & 26.0 & 11.6 & & Irr & 2 \\
\hline 561 & $\ldots$ & B2 V & 203331.68 & +412146.1 & 4.5 & 13.73 & -2.50 & 1.30 & -0.21 & 10.0 & 10.0 & 11.7 & $\mathrm{n}$ & & \\
\hline 568 & $\ldots$ & B3 V & 203333.38 & +410836.3 & 7.0 & 14.76 & -1.90 & 2.16 & -0.18 & 7.6 & 7.6 & 9.6 & $\mathrm{n}$ & & \\
\hline $573 \ldots \ldots$ & $\ldots$ & B3 I & 203333.97 & +41 1938.4 & 5.3 & 13.87 & -6.30 & 1.62 & -0.13 & 22.0 & 23.5 & 14.9 & $\mathrm{n}$ & & \\
\hline $575 \ldots \ldots \ldots \ldots \ldots \ldots$ & B2 V & $\mathrm{B} 2 \mathrm{Ve}$ & 203334.36 & +411811.6 & 5.9 & 13.41 & -2.50 & 1.77 & -0.21 & 10.0 & 10.0 & 9.9 & & & \\
\hline 576 & $\ldots$ & B7: V & 203334.60 & +412137.4 & 4.6 & 14.71 & -1.00 & 1.39 & -0.13 & 4.6 & 4.6 & 10.1 & $\mathrm{n}$ & & \\
\hline 588 & B0 V & $\mathrm{B} 0 \mathrm{~V}$ & 203337.02 & +411611.4 & 5.8 & 12.40 & -3.80 & 1.66 & -0.26 & 17.5 & 17.5 & 10.4 & & Cst & 2 \\
\hline 601 … & O9.5 III & B0 Iab & 203339.14 & +411926.1 & 5.1 & 11.07 & -6.50 & 1.47 & -0.22 & 25.0 & 27.0 & 12.5 & & IS: & 3 \\
\hline $605 \ldots \ldots \ldots \ldots \ldots \ldots \ldots . .$. & B1 V & $\mathrm{B} 1 \mathrm{~V}$ & 203339.84 & +412252.4 & 4.3 & 11.78 & -3.20 & 1.19 & -0.23 & 13.8 & 13.8 & 10.7 & & & \\
\hline 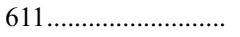 & $\mathrm{O} 7 \mathrm{~V}$ & O7 V & 203340.88 & +413018.5 & 5.5 & 12.77 & -4.63 & 1.55 & -0.29 & 26.5 & 26.5 & 11.8 & & & \\
\hline $620 \ldots \ldots \ldots \ldots \ldots \ldots \ldots .$. & $\ldots$ & $\mathrm{B} 0 \mathrm{~V}$ & 203342.38 & +411145.8 & 6.2 & 13.89 & -3.80 & 1.82 & -0.26 & 17.5 & 17.5 & 11.4 & $\mathrm{n}$ & & \\
\hline $621 \ldots \ldots \ldots \ldots \ldots \ldots .$. & $\ldots$ & B1 V: & 203342.57 & +411456.9 & 6.3 & 14.93 & -3.20 & 1.91 & -0.18 & 13.8 & 13.8 & 11.8 & $\mathrm{n}$ & & \\
\hline $632 \ldots \ldots \ldots \ldots \ldots \ldots \ldots \ldots \ldots$ & O9 I & O9 I & 203346.15 & +413300.5 & 5.6 & 9.88 & -6.29 & 1.59 & -0.27 & 32.0 & 34.5 & 10.5 & & & \\
\hline $635 \ldots \ldots \ldots \ldots \ldots \ldots \ldots$ & $\ldots$ & B1 III & 203346.85 & +410801.9 & 5.8 & 13.81 & -4.30 & 1.72 & -0.21 & 17.5 & 18.3 & 12.3 & $\mathrm{n}$ & & \\
\hline 639.................................. & $\ldots$ & B2 V & 203347.63 & +410906.5 & 6.0 & 14.37 & -2.50 & 1.77 & -0.21 & 10.0 & 10.0 & 10.9 & $\mathrm{n}$ & & \\
\hline 641.... & $\ldots$ & B5 V: & 203347.58 & +412957.7 & 5.0 & 14.27 & -1.35 & 1.51 & -0.15 & 5.9 & 5.9 & 9.3 & $\mathrm{n}$ & & \\
\hline $642 \ldots \ldots \ldots \ldots \ldots \ldots \ldots \ldots \ldots$ & B1 III & B1 III & 203347.88 & +412041.7 & 5.3 & 11.78 & -4.30 & 1.55 & -0.21 & 17.5 & 18.3 & 10.8 & & & \\
\hline $645 \ldots \ldots \ldots \ldots \ldots \ldots$ & $\ldots$ & B2 III & 203348.40 & +41 1314.1 & 6.2 & 14.65 & -3.70 & 1.87 & -0.19 & 14.8 & 15.0 & 12.1 & $\mathrm{n}$ & & \\
\hline 646................................ & B1.5: $\mathrm{V}$ & B1.5 V & 203348.88 & +411940.9 & 4.8 & 13.34 & -2.80 & 1.39 & -0.22 & 11.9 & 11.9 & 11.3 & & & \\
\hline $650 \ldots \ldots \ldots \ldots \ldots \ldots . .$. & $\ldots$ & B2 Ve: & 203348.83 & +413739.7 & 5.2 & 14.94 & -2.50 & 1.53 & -0.21 & 10.0 & 10.0 & 12.2 & $\mathrm{n}$ & & \\
\hline 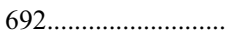 & B0 V & B0 V & 203359.32 & +410538.4 & 5.9 & 13.61 & -3.80 & 1.69 & -0.26 & 17.5 & 17.5 & 11.5 & & & \\
\hline $696 \ldots \ldots \ldots \ldots \ldots \ldots \ldots \ldots$ & $09.5 \mathrm{~V}$ & $09.5 \mathrm{~V}$ & 203359.57 & +411736.1 & 5.8 & 12.32 & -3.90 & 1.65 & -0.27 & 16.5 & 16.5 & 10.4 & & $\mathrm{EW} / \mathrm{KE} ; P=1.46$ days & 4 \\
\hline $712 \ldots \ldots \ldots \ldots \ldots \ldots \ldots$ & $\ldots$ & B1 V & 203404.43 & +410808.4 & 6.1 & 13.66 & -3.20 & 1.80 & -0.23 & 13.8 & 13.8 & 10.7 & $\mathrm{n}$ & & \\
\hline $716 \ldots \ldots \ldots \ldots \ldots \ldots \ldots \ldots$ & $\mathrm{O} 9 \mathrm{~V}$ & O9 V & 203404.95 & +410513.2 & 6.4 & 13.50 & -4.05 & 1.84 & -0.28 & 18.0 & 18.0 & 11.1 & & & \\
\hline
\end{tabular}


TABLE 2-Continued

\begin{tabular}{|c|c|c|c|c|c|c|c|c|c|c|c|c|c|c|c|}
\hline $\begin{array}{l}\text { Star } \\
\text { (MT) } \\
\text { (1) }\end{array}$ & $\begin{array}{l}\text { Lit S.C. } \\
\text { (2) }\end{array}$ & $\begin{array}{c}\text { Our S.C. } \\
\text { (3) }\end{array}$ & $\begin{array}{c}\text { R.A. } \\
\text { (J2000.0) } \\
\text { (4) }\end{array}$ & $\begin{array}{c}\text { Decl. } \\
(\mathrm{J} 2000.0) \\
(5)\end{array}$ & $\begin{array}{l}A_{V} \\
(6)\end{array}$ & $\begin{array}{c}V \\
(7)\end{array}$ & $\begin{array}{l}M_{V} \\
(8)\end{array}$ & $\begin{array}{c}(B-V) \\
(9)\end{array}$ & $\begin{array}{c}(B-V)_{0} \\
\quad(10)\end{array}$ & $\begin{array}{l}M_{\mathrm{PD}} \\
\left(M_{\odot}\right) \\
(11)\end{array}$ & $\begin{array}{c}M_{0} \\
\left(M_{\odot}\right) \\
(12)\end{array}$ & $\begin{array}{l}\text { DM } \\
\text { (13) }\end{array}$ & $\begin{array}{c}\text { Notes } \\
(14)\end{array}$ & $\begin{array}{c}\text { Ph. Activ. } \\
\text { (15) }\end{array}$ & $\begin{array}{l}\text { Ref. } \\
\text { (16) }\end{array}$ \\
\hline 720 & B: & $09.5 \mathrm{~V}$ & 203406.10 & +410809.6 & 7.0 & 13.59 & -3.90 & 2.05 & -0.27 & 16.5 & 16.5 & 10.5 & & & \\
\hline 734 & O5 I & O5 I & 203408.54 & +413659.3 & 5.4 & 10.03 & -6.33 & 1.49 & -0.32 & 50.9 & 53.0 & 10.9 & & Susp & 1 \\
\hline $736 \ldots \ldots \ldots \ldots \ldots \ldots$ & O9 V & $\mathrm{O} 9 \mathrm{~V}$ & 203409.52 & +413413.4 & 5.2 & 12.79 & -4.05 & 1.46 & -0.28 & 18.0 & 18.0 & 11.6 & & & \\
\hline $745 \ldots \ldots \ldots \ldots \ldots \ldots \ldots \ldots \ldots$ & $\mathrm{O} 7 \mathrm{~V}$ & $\mathrm{O} 7 \mathrm{~V}$ & 203413.50 & +413502.6 & 5.4 & 11.91 & -4.63 & 1.50 & -0.29 & 26.5 & 26.5 & 11.1 & & & \\
\hline 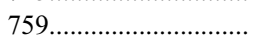 & $\ldots$ & $\mathrm{B} 1 \mathrm{~V}$ & 203424.56 & +412624.7 & 5.7 & 14.65 & -3.20 & 1.67 & -0.23 & 13.8 & 13.8 & 12.1 & $\mathrm{n}$ & & \\
\hline $771 \ldots$ & $\mathrm{O} 7 \mathrm{~V}$ & $\mathrm{O} 7 \mathrm{~V}$ & 203429.52 & +413145.5 & 7.0 & 12.06 & -4.63 & 2.05 & -0.29 & 26.5 & 26.5 & 9.6 & & & \\
\hline 793 & B1.5 III & B2 IIIe & 203443.51 & +412904.8 & 5.2 & 12.29 & -3.70 & 1.54 & -0.19 & 14.8 & 15.0 & 10.7 & & & \\
\hline
\end{tabular}

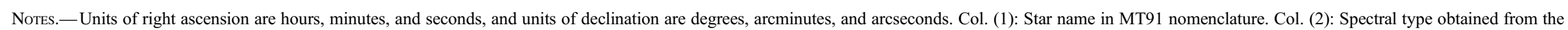

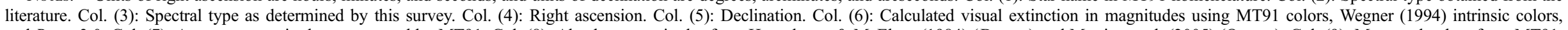

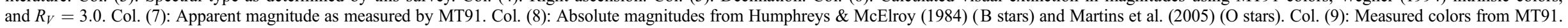

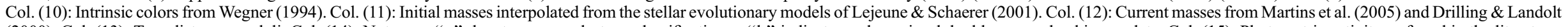

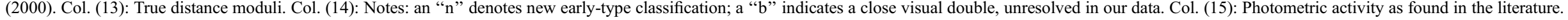
Col. (16): Literature reference for photometric activity. Table 2 is also available in machine-readable form in the electronic edition of the Astrophysical Journal.

References.-(1) Kukarkin \& Kholopov 1982; (2) Pigulski \& Kolaczkowski 1998; (3) Romano 1969; (4) Rios \& DeGioia-Eastwood 2004. 
TABLE 3

NON-OB STARS

\begin{tabular}{|c|c|c|c|c|}
\hline $\begin{array}{c}\text { Star } \\
(\mathrm{MT}) \\
(1)\end{array}$ & $\begin{array}{c}\text { R.A. } \\
(\mathrm{J} 2000.0) \\
(2)\end{array}$ & $\begin{array}{c}\text { Decl. } \\
(\mathrm{J} 2000.0) \\
(3)\end{array}$ & $\begin{array}{c}V \\
(4)\end{array}$ & $\begin{array}{l}\text { S.C. } \\
\text { (5) }\end{array}$ \\
\hline 34.................... & 203054.41 & +413249.3 & 15.44 & G? \\
\hline $35 \ldots \ldots \ldots \ldots$ & 203054.94 & +413545.6 & 15.63 & $\mathrm{G}-\mathrm{K}$ \\
\hline $50 \ldots \ldots \ldots \ldots \ldots . . .$. & 203105.61 & +413430.7 & 14.33 & G \\
\hline $52 \ldots \ldots \ldots \ldots \ldots . . .$. & 203108.25 & +413532.3 & 10.98 & $\mathrm{~F}$ \\
\hline $54 \ldots \ldots \ldots \ldots \ldots$ & 203108.33 & +413537.1 & 11.26 & G \\
\hline $62 \ldots \ldots \ldots \ldots$ & 203111.79 & +413424.5 & 14.76 & G \\
\hline $100 \ldots \ldots \ldots \ldots$ & 203132.44 & +413741.3 & 15.60 & $\mathrm{G}$ \\
\hline 107.................. & 203134.05 & +413102.6 & 14.80 & $\mathrm{G} ?$ \\
\hline $118 \ldots \ldots \ldots \ldots$ & 203138.59 & +411953.8 & 14.29 & $\mathrm{~F}$ ? \\
\hline $126 \ldots \ldots \ldots \ldots . . .$. & 203141.49 & +412303.7 & 15.02 & G \\
\hline $133 \ldots \ldots \ldots \ldots$ & 203142.68 & +412954.5 & 15.04 & $\mathrm{G}$ ? \\
\hline 137................... & 203144.89 & +412138.1 & 14.47 & $\mathrm{G}$ \\
\hline $141 \ldots \ldots \ldots \ldots$ & 203145.91 & +413449.2 & 15.36 & G \\
\hline $156 \ldots \ldots \ldots \ldots$ & 203152.64 & +412535.8 & 15.37 & G \\
\hline $235 \ldots \ldots \ldots \ldots . . .$. & $2032 \quad 19.81$ & +412351.5 & 13.99 & G \\
\hline $242 \ldots \ldots \ldots \ldots$ & 203223.57 & +411924.3 & 15.36 & G \\
\hline $244 \ldots \ldots \ldots \ldots . .$. & 203223.83 & +411936.2 & 15.08 & A \\
\hline $267 \ldots \ldots \ldots \ldots$ & 203231.45 & +411408.8 & 12.87 & G \\
\hline $272 \ldots \ldots \ldots \ldots$ & 203232.39 & +413237.3 & 15.01 & G \\
\hline $279 \ldots \ldots \ldots \ldots . . .$. & 203234.33 & +410426.0 & 13.85 & $\mathrm{~A}$ \\
\hline $289 \ldots \ldots \ldots \ldots$ & 203235.79 & +413537.8 & 14.71 & A \\
\hline $308 \ldots \ldots \ldots \ldots . . . . .$. & 203241.37 & +413026.7 & 15.12 & G \\
\hline $398 \ldots \ldots \ldots \ldots . . .$. & 203304.40 & +413255.8 & 14.70 & G \\
\hline $430 \ldots \ldots \ldots \ldots$ & 203310.76 & +410720.5 & 14.41 & $\mathrm{G}$ \\
\hline 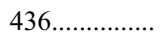 & $2033 \quad 11.07$ & +411445.6 & 16.08 & K \\
\hline $438 \ldots \ldots \ldots \ldots . .$. & $2033 \quad 10.69$ & +413952.9 & 14.75 & $\mathrm{~K}$ \\
\hline 463................. & $2033 \quad 14.86$ & +411934.7 & 15.01 & $\mathrm{~F}$ \\
\hline $519 \ldots \ldots \ldots \ldots$ & 203323.85 & +413037.8 & 15.06 & G \\
\hline $524 \ldots \ldots \ldots \ldots$ & 203324.70 & +414059.3 & 13.06 & A \\
\hline $526 \ldots \ldots \ldots \ldots$ & $2033 \quad 25.37$ & +413028.1 & 14.59 & M \\
\hline 617.................... & 203342.15 & +412222.8 & 14.93 & Late? \\
\hline 637................. & 203346.58 & +413834.3 & 15.00 & G \\
\hline $665 \ldots \ldots \ldots \ldots$ & 203353.65 & +412532.3 & 14.76 & $\mathrm{~K}$ \\
\hline $728 \ldots \ldots \ldots \ldots . . .$. & 203406.64 & +414313.3 & 15.15 & $\mathrm{~K}$ \\
\hline $735 \ldots \ldots \ldots$ & 203409.47 & +412943.9 & 15.38 & $\mathrm{~F}$ \\
\hline 737.................. & 203409.85 & +412557.5 & 14.74 & $\mathrm{G}$ \\
\hline $789 \ldots \ldots \ldots \ldots$ & 203439.49 & +413746.2 & 11.63 & Late \\
\hline
\end{tabular}

Notes.-Units of right ascension are hours, minutes, and seconds, and units of declination are degrees, arcminutes, and arcseconds. Col. (1): Star name in MT91 nomenclature. Col. (2): Right ascension. Col. (3): Declination. Col. (4): Apparent magnitude as measured by MT91. Col. (5): Spectral type.

this section of the spectrum and the Keck spectrum showed no evidence of them. The Lick data did cover this spectral range but were not of sufficient $\mathrm{S} / \mathrm{N}$ for detailed classification.

\subsection{Visual Extinction and Distance}

Table 2 provides the calculated visual extinctions (col. [6]) and distance moduli (col. [13]) for the 146 OB stars in the direction of Cyg OB2. The values were calculated from apparent visual magnitudes (col. [7]), absolute visual magnitudes (col. [8]), colors $(B-V)$ (col. [9]), and intrinsic colors $(B-V)_{0}$ (col. [10]). The apparent visual magnitudes and $(B-V)$ colors were obtained from MT91. The absolute visual luminosities were adopted from Martins et al. (2005) for the $\mathrm{O}$ stars and Humphreys \& McElroy (1984) for the B stars. The intrinsic $(B-V)_{0}$ colors were based on adopted spectral types and obtained from Wegner (1994).

We computed the visual extinctions, $A_{V}$, using

$$
A_{V}=R_{V}\left[(B-V)-(B-V)_{0}\right]
$$

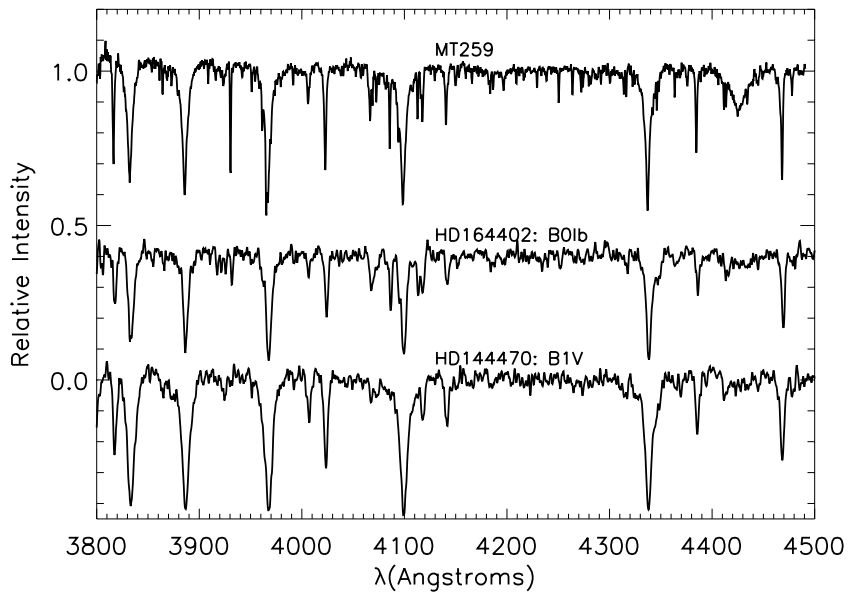

FIG. 1.-2001 September 9 WIYN spectrum of MT 259 (Cyg OB2 No. 21) and comparison spectra from Walborn \& Fitzpatrick (1990). MT 259 is more consistent with a B0 Ib classification.

where $R_{V}$ is the ratio of total to selective extinction. We adopt $R_{V}=3.0$ based on the studies of Hanson (2003) and MT91. Visual extinctions range between $A_{V}=3.5$ and 10.4 (for No. 12) with a mean near $A_{V}=5.4 \mathrm{mag}$, consistent with prior results (e.g., MT91). Figure 4 shows an extinction map for all OB stars in the direction of Cyg OB2, where the relative symbol size is proportional to $A_{V}$. Using these calculated extinctions, we computed photometric distance moduli to all of the OB stars using

$$
\mathrm{DM}=V-M_{V}-A_{V},
$$

where $V$ is the apparent visual magnitude and $M_{V}$ is the absolute visual magnitude. Figure 5 shows a histogram of the computed distance moduli for all $146 \mathrm{OB}$ stars in the direction of Cyg OB2. This histogram peaks at $\mathrm{DM} \simeq 11.3 \mathrm{mag}$ or $\simeq 1.8 \mathrm{kpc}$, in good agreement with the commonly adopted distance estimate of 1.7 kpc (Hanson 2003; MT91; Schulte 1958). The distribution is approximately Gaussian with a width of $\sigma=1.0 \mathrm{mag}$. The large width reflects the uncertainties on the absolute visual magnitudes, particularly for evolved stars, and uncertainties in temperature and luminosity classification. Hanson (2003) found a similar scatter in DM of up to $1.5 \mathrm{mag}$ and mean distance moduli from 10.08 to $11.16 \mathrm{mag}$, depending on the adopted absolute magnitude calibrations. An additional systematic uncertainty arises from the presence of unresolved binaries, which lead to smaller inferred distance moduli. Unresolved binaries are at least partially responsible for the asymmetry in the distribution in Figure 5. A third source of uncertainty might also stem from the ratio of total to selective extinction, $R_{V}$. Patriarchi et al. (2003) found an average $R_{V}=3.1$ for the Cygnus region, but a spread of $\sim 1$. A spread of 1 in $R_{V}$ translates to a spread in $A_{V}$ of approximately 1-2 mag for our sample. The compound extinction components in combination with the density of documented O stars in Cyg OB2 could create conditions under which the dust grain size distribution and composition vary and lead to a variable $R_{V}$ across the cluster.

\subsection{New Early Types and Cluster Membership}

The true distance moduli shown in Figure 5 are listed in column (13) of Table 2. For the purposes of this study, we accept as provisional members all stars with distance moduli between $\sim 8.5$ and 14.5 mag that lie within the broad Gaussian distribution. Three stars fall outside of the distribution (MT 427, MT 573, and MT 304). It will be shown in the following section that 

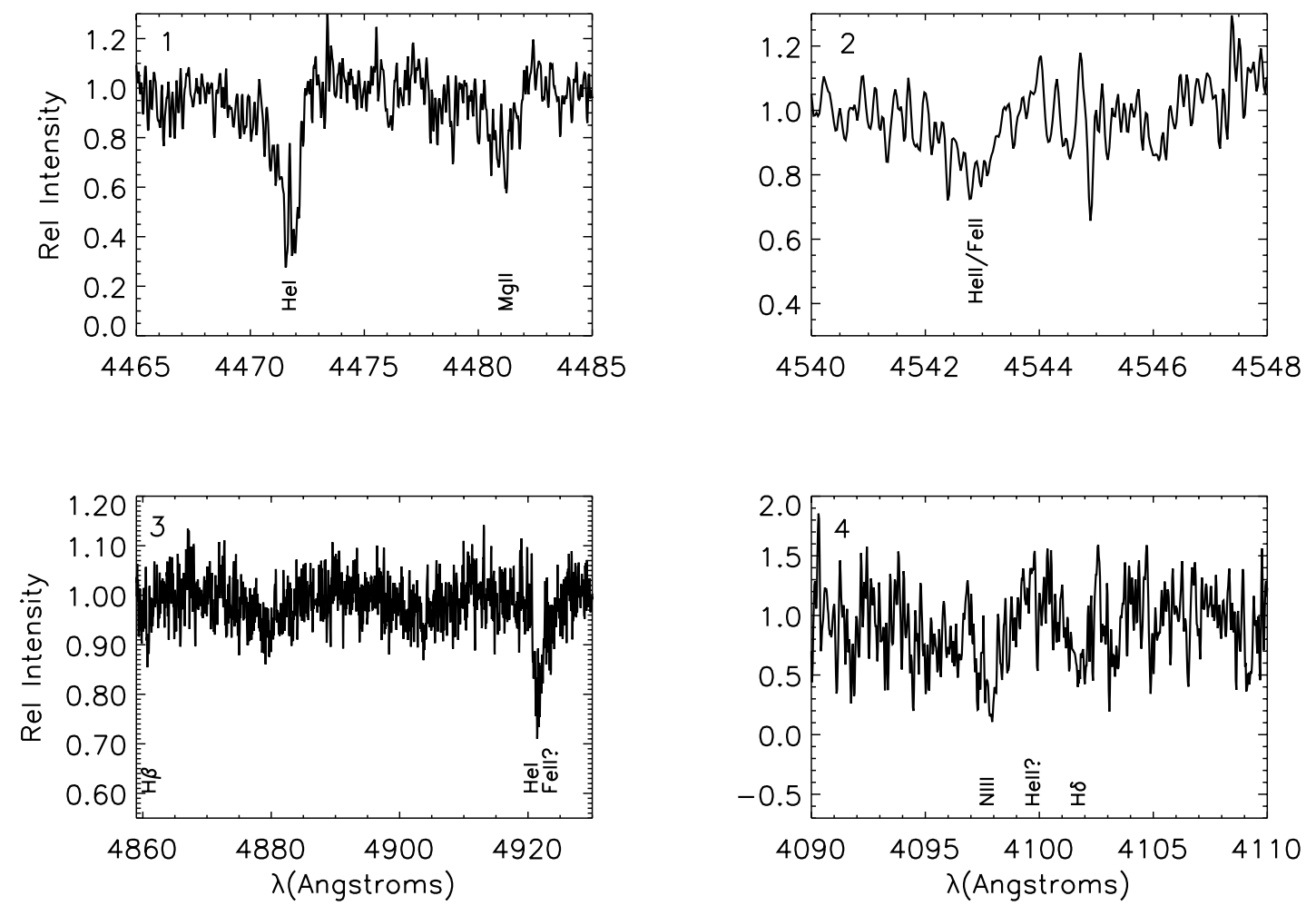

FIG. 2. - Close-up of portions of MT 304 (Cyg OB2 No. 12) from the 2000 September 18 spectrum taken at Keck. Top left: Important He I $\lambda 4471 / \mathrm{Mg}$ II $\lambda 4481$ ratio of approximately $2: 1$. Top right: $\mathrm{He}_{\text {II }} / \mathrm{Fe}$ I blend at $4542 \AA$, very weak in early B stars, and absent in later types. Bottom left: He I $\lambda 4922$ and an $\mathrm{H} \beta$ line filled in by emission. The strength of $\lambda 4922$ and the weak or absent Fe II indicate a type of B5 or earlier. Bottom right: Emission-filled H $\delta, \mathrm{N}$ III $\lambda 4097$, and possible He II $\lambda 4100$.

including all such stars does not significantly affect the computed slope of the IMF. About half of these were previously identified spectroscopically as probable members by MT91. The other probable members were determined photometrically as a group by MT91 but not named individually. The total sample consists of 108 main-sequence stars, 36 evolved stars, and two with indeterminate luminosity class. We have identified 73 early-type stars without previous spectral classification in the literature. Newly classified early-type stars are denoted in column (14) of Table 2 with an "n." Of these, 56 are main-sequence stars, mostly type B0 and later.
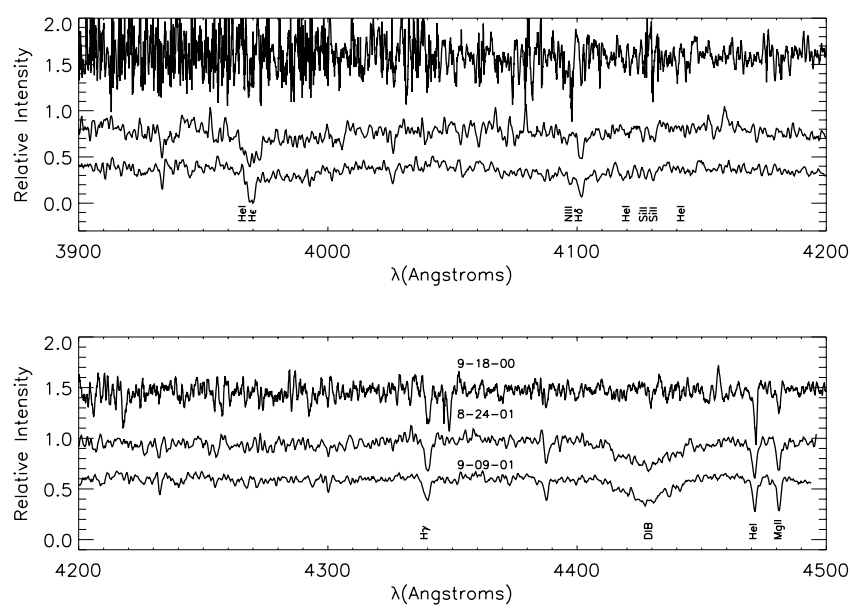

FIG. 3.-Comparison of the spectra for MT 304 (Cyg OB2 No. 12) obtained over 1 yr: Keck 2000 September 18 (smoothed by $3 \AA$ ) followed by WIYN 2001 August and September. Changes in Balmer line equivalent width, the N III $\lambda 4097$ depth, and the He I $\lambda 4471 / \mathrm{Mg}_{\mathrm{I}} \lambda 4481$ ratios are also shown. The sequence shows an evolution from B3 I in 2000 September to at least B8 I in 2001 September.
As a secondary means of assessing membership for the earlytype stars, we examined the equivalent widths of the diffuse interstellar band (DIB) absorption feature at $4428 \AA$. We found that nearly all of the newly identified stars had $\mathrm{EW}_{\mathrm{DIB}}=3 \pm 1 \AA$, consistent with the established association members (Snow et al. 2002). Given the large spread in reddening and DIB equivalent width for Cyg OB2, as well as the fact that Snow et al. (2002) found a poor correlation between reddening and DIB strength for this cluster, we conclude that this relation is not a useful discriminant of members from field stars.

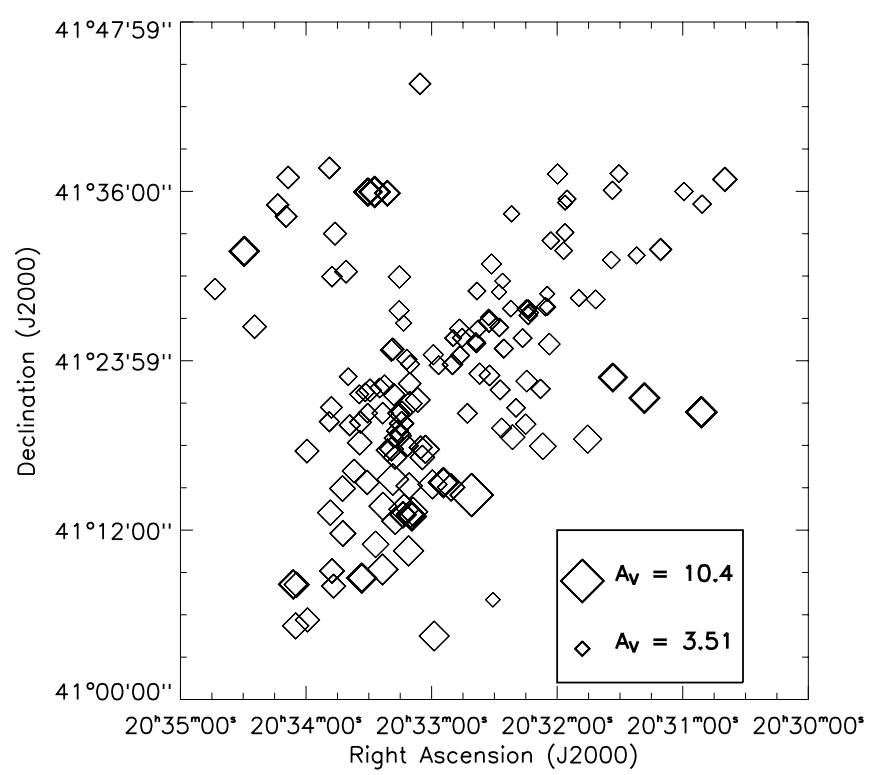

FIG. 4.- Map of calculated extinction, $A_{V}$, for $146 \mathrm{OB}$ stars in the direction of Cyg OB2. The relative size of the symbol is proportional to $A_{V}$. 


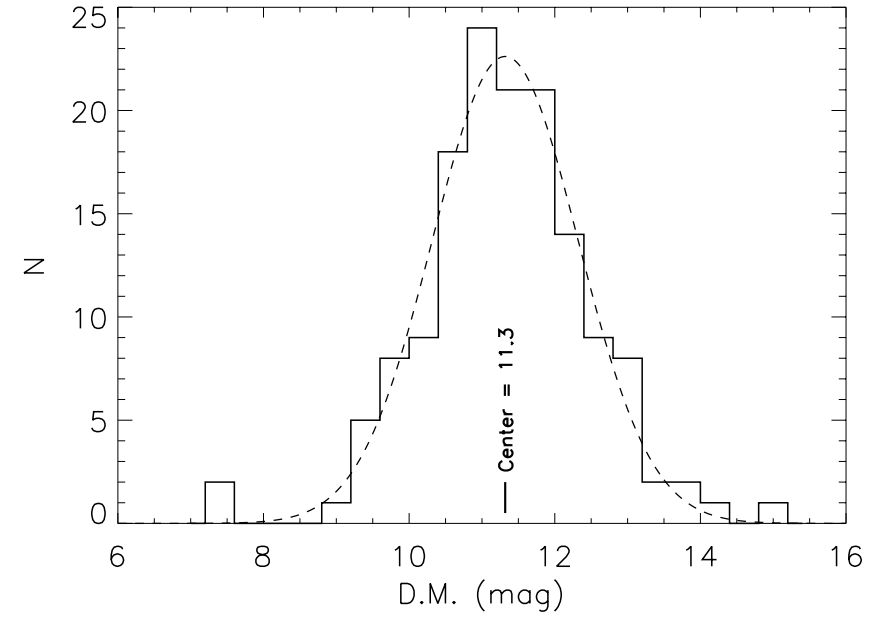

FIG. 5.- Histogram of spectrophotometric distances to the 146 OB stars in the direction of Cyg OB2. The mean distance modulus is 11.3 mag or $\sim 1.8 \mathrm{kpc}$. The approximately Gaussian dispersion is due to uncertainties in the absolute magnitudes, particularly of the post-main-sequence stars. We adopt all stars with distance moduli between 8.5 and 14.5 as provisional members of the association while acknowledging that this generous range includes some foreground and background objects.

\subsection{Initial Mass Function}

Studies of the slope of the Cyg OB2 IMF have reached disparate conclusions. K00 used JHK Two Micron All Sky Survey Point Source Catalog (Skrutski et al. 2006) photometry and a $K$-band mass-luminosity relation to calculate a mass function slope of $\Gamma=-1.6 \pm 0.1$. This is steeper than previous studies of Cyg OB2. MT91 utilized the evolutionary models of Maeder \& Meynet (1988) with an H-R diagram constructed from their spectroscopy and "best" photometry to obtain a slope of $\Gamma=$ $-1.0 \pm 0.1$. This is relatively shallow compared to the canonical Salpeter (1955) value of $\Gamma=-1.35$. Massey et al. (1995) found a similar slope of $\Gamma=-0.9 \pm 0.2$, using the same technique over a mass range of $15-25 M_{\odot}$. In both calculations, a coeval system was assumed.

We calculated an IMF slope using the spectroscopic masses for the previously classified 73 OB stars and the 73 newly classified OB stars reported here. Present-day masses for each star were taken from Martins et al. (2005) for the O stars and interpolated from the tables of Drilling \& Landolt (2000) for the B stars. Initial masses were also estimated for each of the evolved stars from the Lejeune \& Schaerer (2001) stellar evolutionary models. Table 2 lists the present-day and adopted initial masses for each star in columns (11) and (12). Figure 6 shows the cumulative logarithmic mass distribution of 143 Cyg OB2 stars with spectroscopic masses (minus Cyg OB2 No. 12 owing to the uncertainty in its spectral type, mass-loss rate, and current mass). We use a cumulative rather than a differential mass distribution to measure the IMF slope in order to mitigate uncertainties caused by discrete mass bins and the choice of bin size and placement. Diamonds indicate the logarithmic number of stars with a logarithmic mass greater than or equal to that point. The error bars reflect Poisson statistics. It is clear from the change in slope at $\log$ (mass) $\simeq 1.0$ that the spectroscopic survey becomes incomplete at masses below $\sim 15 M_{\odot}$, corresponding approximately to a B1 V star. The solid line represents a linear fit to all points greater than this cutoff. We obtain a slope of $\Gamma=-2.2 \pm 0.1$. This is much steeper than the canonical Salpeter (1955) value of $\Gamma=-1.35$. This value is also steeper than the results of $\mathrm{K} 00$ and significantly more so than MT91. The possible inclusion of

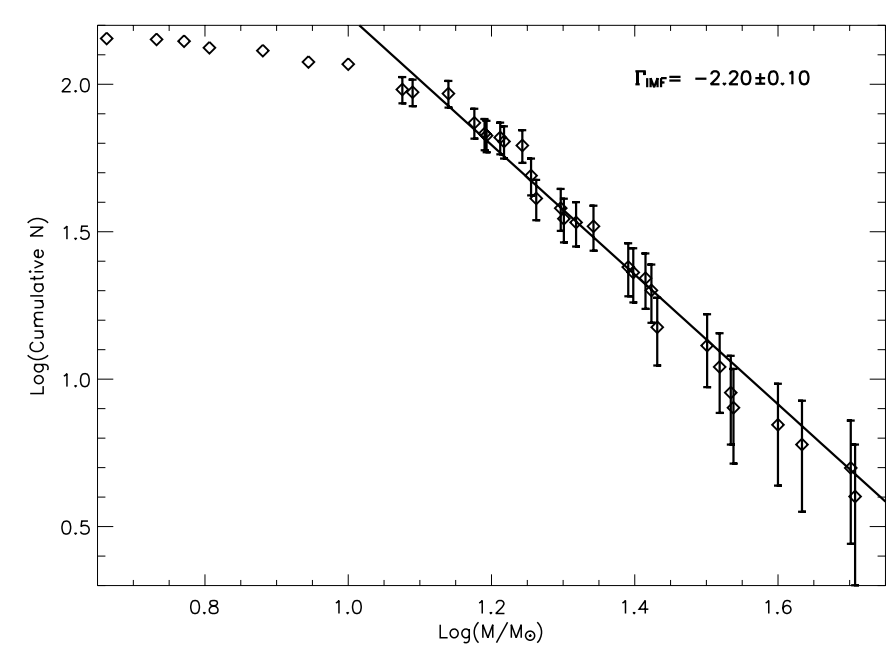

FIG. 6.-Plot of mass distribution for provisional members of Cyg OB2. The IMF is calculated using a cumulative mass count. The solid line is a linear fit to masses greater than $\log \left(M / M_{\odot}\right)=1.0$. A slope of $\Gamma=-2.2 \pm 0.1$ has been calculated. Limiting the analysis to stars having distance moduli within 1.5 mag of the mean does not change the slope by more than 0.04 .

foreground or background stars may bias the slope toward steeper values. However, the IMF slope does not vary by more than 0.04 from the nominal value of $\Gamma=-2.20$ when we remove stars that lie farther than $1.5 \sigma$ (24 stars) from the mean distance modulus of 11.3. The dominant explanation for the difference between our IMF and the previous results is a systematic difference in the predicted initial stellar masses between the models of Maeder \& Meynet (1988; used by MT91) and Lejeune \& Schaerer (2001) used in this study. The implied masses of Lejeune \& Schaerer (2001) are systematically lower for a given bolometric luminosity and effective temperature compared to the models of Maeder \& Meynet (1988). Effectively this means that we have fewer high-mass stars than MT91, which results in a steeper slope. We also calculated the slope of the present-day mass function (PDMF) to be $\Gamma=-2.3 \pm 0.1$, which is more appropriate for comparison to the photometric PDMF results of K00. It should also be noted that our sample is composed only of stars within the core of Cyg OB2, while the K00 sample encompasses a large number of stars outside the core region.

\section{CALCULATING THE RELATIVE RADIAL VELOCITIES}

We used two methods to calculate the relative and absolute radial velocities: Gaussian profile fitting and cross-correlation. For early-type stars that have a small number of broad spectral features, profile fitting can be a reasonable approach. This method entailed fitting Gaussian profiles to the strong $\mathrm{H}$ and He absorption lines $\left(\mathrm{H} \beta, \mathrm{H} \gamma, \mathrm{H} \delta, \mathrm{He}_{\mathrm{I}}\right.$ 24471, and $\mathrm{He}_{\mathrm{I}}$ 24388). We used a five-parameter Gaussian ${ }^{8}$ of the form

$$
f(x)=A_{0} e^{-(1 / 2)\left[\left(x-A_{1}\right) / A_{2}\right]^{2}}+A_{4} x+A_{3},
$$

where $A_{0}$ is the depth, $A_{1}$ is the center, $A_{2}$ is the FWHM, $A_{3}$ is the constant term, and $A_{4}$ is the linear term. The code performs initial fits to the $\mathrm{H} \beta$ and $\mathrm{He}$ I $\lambda 4471$ lines of a template spectrum (usually the highest quality Keck spectrum) in order to measure the widths of the $\mathrm{H}$ and He lines, respectively. The width parameter, $A_{2}$, for the $\mathrm{H}$ and $\mathrm{He}$ lines is subsequently held constant

8 The IDL package "MPFIT" written by Craig B. Markwardt, NASA GSFC code 662 , is substituted for the standard "GAUSSFIT" and "CURVEFIT" due to its greater control over the fitting parameters. 
TABLE 4

Complete Table of Observations and Radial Velocities

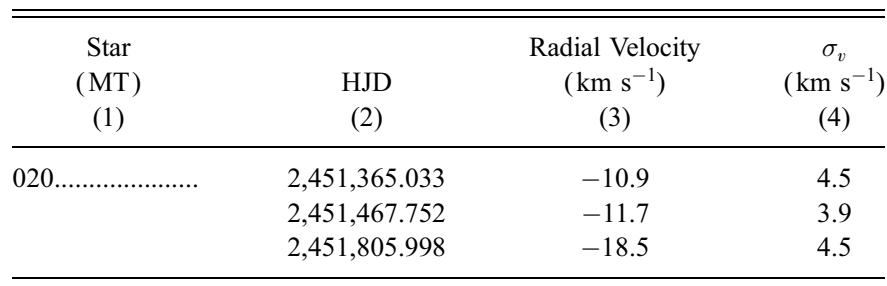

Notes.—Col. (1): Star name in MT91 nomenclature. Col. (2): Heliocentric Julian date. Col. (3): Heliocentric radial velocity. Col. (4): $1 \sigma$ uncertainty. Table 4 is published in its entirety in the electronic edition of the Astrophysical Journal. A portion is shown here for guidance regarding its form and content.

at these initial values. The line centers are fixed relative to each other using the rest wavelengths from atomic line lists (Cowley et al. 2000). The code then fits profiles to all of the $\mathrm{H}$ and $\mathrm{He}$ lines with $A_{0}, A_{3}$, and $A_{4}$ as free parameters for each line. The bestfit parameters are then saved and used to construct a "template" consisting of five Gaussian components. This template is then stepped through a range of velocities from -160 to $160 \mathrm{~km} \mathrm{~s}^{-1}$ at a step size of $1 \mathrm{~km} \mathrm{~s}^{-1}$ to minimize the $\chi^{2}$ between the template and spectra from each epoch. At each velocity step, $A_{0}, A_{3}$, and $A_{4}$ of each component are allowed to vary separately to achieve the best fit. The velocity of the global $\chi^{2}$ minimum is adopted as the most probable velocity for each epoch. Because the $\mathrm{H}$ lines are stronger than the He lines, they were given more weight in the calculation of the total $\chi^{2}$. Spectra from WIYN have limited wavelength coverage and do not include $\mathrm{H} \beta$, so only four Gaussian components are fitted. Spectra from WIRO have limited wavelength coverage and do not include $\mathrm{H} \gamma$; hence, only four Gaussian components are fitted. Velocity uncertainties were calculated using the $\Delta \chi^{2}$ statistic (Press et al. 1992).

Gaussian fitting proved simple to automate for a large data set. We also found it to be more robust for low-S/N spectra and in regions at the edges of spectral orders in the echelle data. However, it often yielded larger uncertainties. We found that in the case of poor-quality spectra the fitting routine would provide solutions for local or no minima.

We also measured radial velocities with cross-correlation techniques using the IRAF xcsao task, which is part of the RVSAO package (Kurtz et al. 1992). This method had the advantage of using information in all spectral features, although for mainsequence stars most of the cross-correlation power stems from the few strong $\mathrm{H}$ and $\mathrm{He}$ lines. As the template for cross-correlation we tried using both an observed spectrum (usually a high-quality Keck spectrum) and a model stellar atmosphere (Lanz \& Hubeny 2003; TLUSTY) of the appropriate effective temperature and gravity. The model atmosphere is not rotationally broadened to match individual stars in our sample, but in the vast majority of cases the model line widths appear well matched to the spectra. In most cases, the analysis using atmospheric model templates produced smaller velocity uncertainties than either the results from Gaussian fitting or cross-correlation with observed spectral templates. The smaller uncertainties result from the higher $\mathrm{S} / \mathrm{N}$ of the model templates and the fact that this method uses the power of many additional spectral features in the templates and data to constrain radial velocities. Varying the gravity and effective temperature of the template produced little or no change in the correlation results. Therefore, we adopt the velocities obtained by cross-correlation with the model atmospheres. Table 4 contains the star name, heliocentric Julian date, relative radial velocities, and $1 \sigma$ error estimates for each epoch of observation

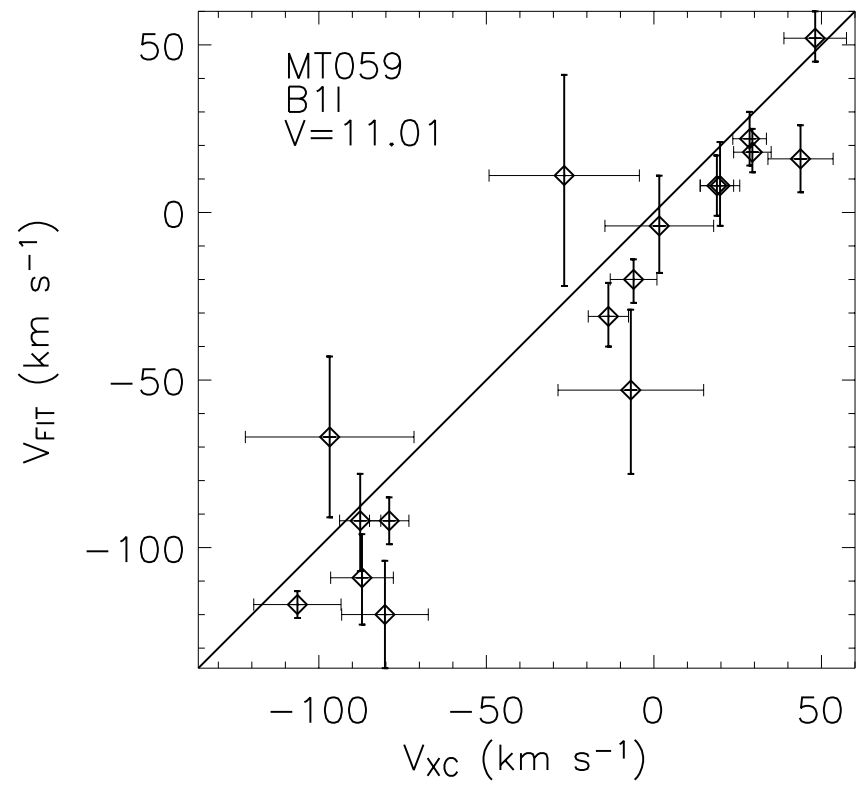

FIG. 7.-Comparison of relative radial velocity results for Gaussian profile fitting vs. cross-correlation techniques for MT 059 . A minor offset of $\sim 10-15 \mathrm{~km} \mathrm{~s}^{-1}$ is observed in most comparisons, most likely attributed to the use of a model atmosphere as a template for the cross-correlation. On average, the larger errors belong to the Gaussian profile fitting.

in columns (1)-(4), respectively. The uncertainties are calculated within xcsao using the equation

$$
\sigma_{v}=\frac{3 w}{8(1+r)}
$$

where $w$ is the FWHM of the correlation peak and $r$ is the ratio of the correlation peak height to the amplitude of antisymmetric noise (Kurtz et al. 1992).

We found that the resultant relative radial velocities and their uncertainties showed good agreement between the two analysis methods. Figures 7-10 show comparisons of velocities and their

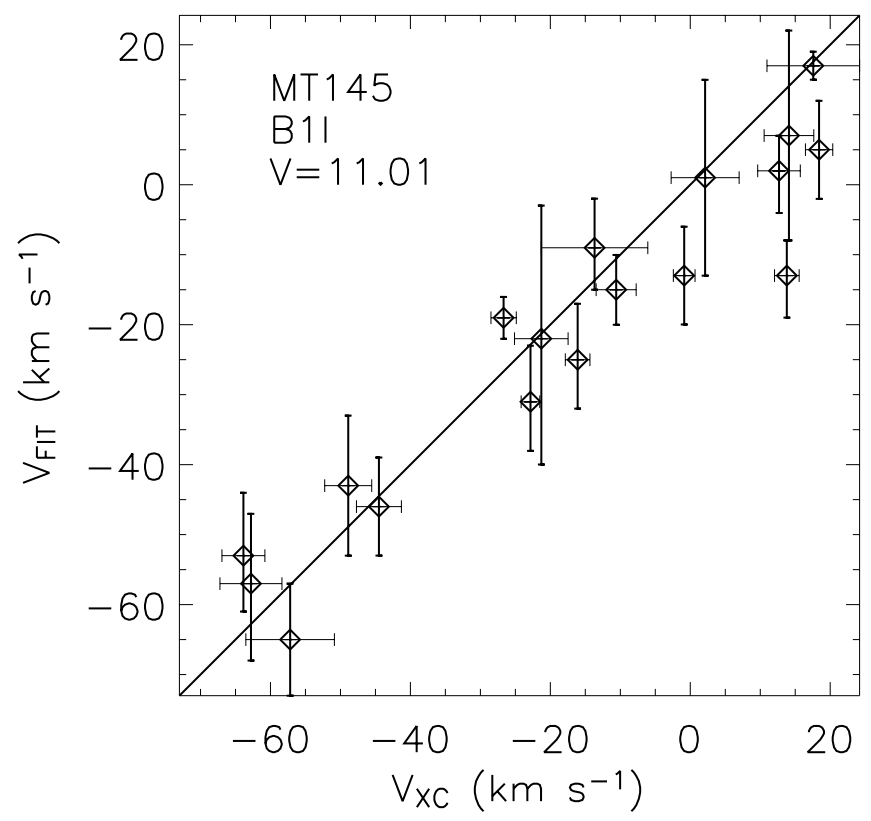

FIG. 8. Comparison of relative radial velocity results for Gaussian profile fitting and cross-correlation techniques for MT 145. 


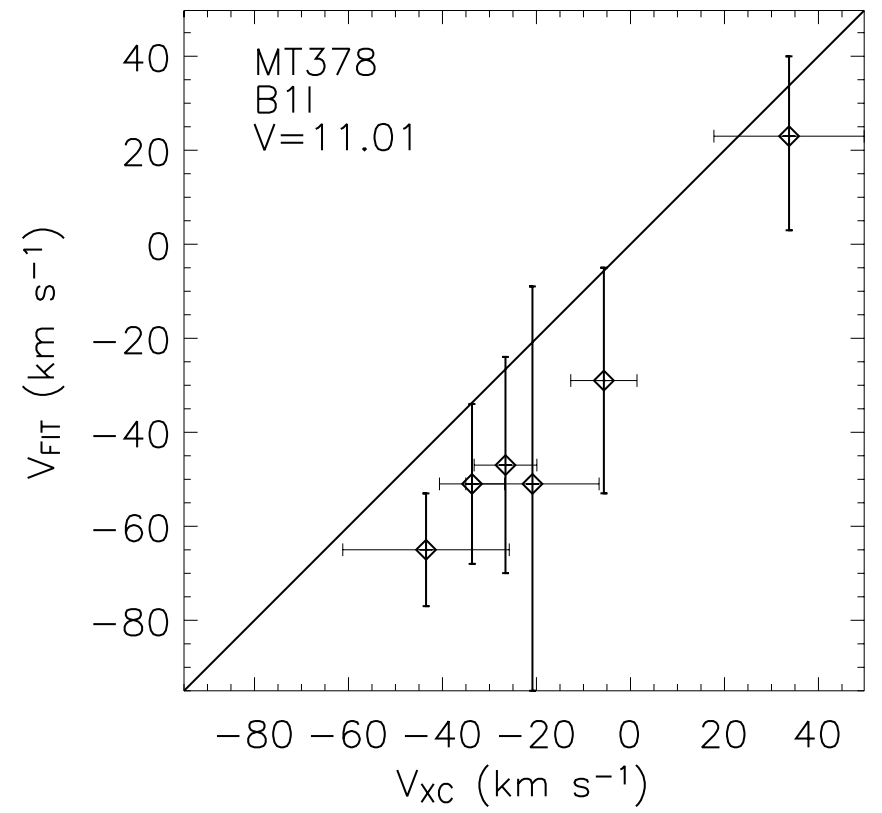

FIG. 9.-Comparison of relative radial velocity results for Gaussian profile fitting and cross-correlation techniques for MT 378.

uncertainties for an O8 V, O9 III, B0 V, and B1 I star, respectively. These figures demonstrate that there is a strong correlation between velocities obtained with the fitting and the cross-correlation techniques. Frequently, the error bars are smaller for the crosscorrelation analysis, especially for evolved stars that have more spectral features that are utilized by cross-correlation methods but not by our Gaussian fitting code. Deviations from the 1:1 correspondence are generally consistent with the shown error, indicating that the statistical uncertainties are well estimated. In most cases, there is also a zero-point offset of $\sim 10-15 \mathrm{~km} \mathrm{~s}^{-1}$ in the sense that the profile-fitted velocities are smaller (i.e., more negative) than the results from cross-correlation. The magnitude of this offset is similar for both main-sequence and evolved stars. Such a modest offset is not unexpected given that the line centers of the model atmospheres should not necessarily agree with the rest wavelengths of the five $\mathrm{H}$ and $\mathrm{He}$ lines used by our profile fitting code.

From the initial sample of 146 possible Cyg OB2 stars, we discarded 26 stars from the sample that had fewer than three observations, poor-quality spectra, or emission lines that interfered with the cross-correlation analysis. The stars showing emission were discarded because the phenomenon might be time variable and produce apparent radial velocity variations that mimic the effects of orbital motion. Furthermore, we eliminated a small number ( $\sim 20$ out of 1139$)$ of individual spectra with low S/N (mostly Lick data from a run plagued by clouds). The remaining sample used for velocity analysis consists of 120 stars.

For the remaining 120 objects, Table 5 lists the identification for each star based on MT91 notation (col. [1]), the spectral type (col. [2]), the number of observations for each star (col. [3]), $V_{\text {avg }}$, the weighted average heliocentric velocity (col. [4]), $V_{\text {mid }} \equiv$ $0.5\left(V_{\max }+V_{\min }\right)$, the average of the largest and smallest observed heliocentric velocities (col. [5]), $V_{h}=0.5\left(V_{\max }-V_{\min }\right)$, a measure of the velocity semiamplitude (col. [6]), $V_{\text {rms }}$, the velocity dispersion (col. [7]), and $\bar{\sigma}_{v}$, the mean velocity uncertainty (col. [8]). $V_{\text {avg }}$ and $V_{\text {mid }}$ are both measures of the systemic velocity for the star, although both are susceptible in different ways to sampling effects and measurement errors. $V_{\text {avg }}$ would provide a robust measure of the true systemic velocity if the observations

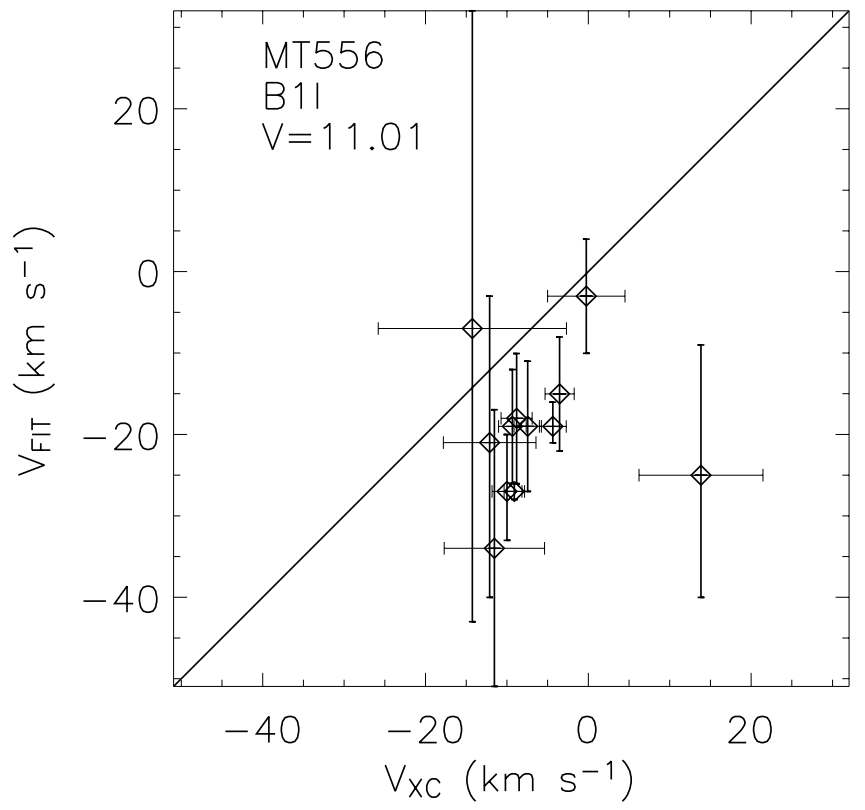

FIG. 10.-Comparison of relative radial velocity results for Gaussian profile fitting and cross-correlation techniques for MT 556.

uniformly sample all orbital phases in a binary system with zero eccentricity. However, our sparse sampling, coupled with the likelihood that some orbits are eccentric, renders this measure less than ideal. $V_{\text {mid }}$ provides an alternative measure of the systemic velocity, which is less susceptible to undersampling but may be more prone to measurement uncertainty. We use both $V_{\text {avg }}$ and $V_{\text {mid }}$ as indicators of the systemic velocities, and we find that they yield comparable results. $V_{h}$ and $V_{\text {rms }}$ are both measures of the observed velocity variations for a given stellar system. $V_{h}$ is a measure of the velocity semiamplitude of the system, albeit an imperfect one, when the velocity curve is not sampled at all phases. $V_{\mathrm{rms}}$ is another measurement that reflects the level of velocity variations in a system. We use $\bar{\sigma}_{v}$ to describe a characteristic uncertainty averaged over all observations.

There is an additional source of random and/or systematic uncertainty that may contribute to the overall error budget of each measurement. Stellar photospheric line profile variations may be present among some of the most massive stars, especially the evolved stars in our sample ( 31 of 120 stars, or about $\sim 26 \%$, are post-main-sequence stars). Line profile variations attributed to atmospheric pulsations are observed in $\geq 77 \%$ of evolved O stars and in some Be stars (Penrod 1986; Vogt \& Penrod 1983) but rarely among dwarf stars (Fullerton et al. 1996). These phenomena could mimic the effects of bona fide orbital velocity variability. Irregular variability due to these effects might also mask a true low-amplitude binary. More frequent time sampling of candidate variables is the only way to identify bona fide binaries and reduce misidentification.

\section{RESULTS}

\subsection{Velocity Variations}

Figure 11 shows a histogram of the observed radial velocities and mean uncertainties. This figure illustrates the distribution of velocity dispersions, $V_{\text {rms }}$, calculated from the multiple measurements of the $120 \mathrm{OB}$ stars (solid line) along with the distribution of mean velocity uncertainties, $\bar{\sigma}_{v}$ (dashed line). The dotted line shows the distribution of $V_{h}$. The lowest velocity bin from 0 to $5 \mathrm{~km} \mathrm{~s}^{-1}$ is sparsely populated because observational errors 
TABLE 5

Derived Radial Velocity Parameters

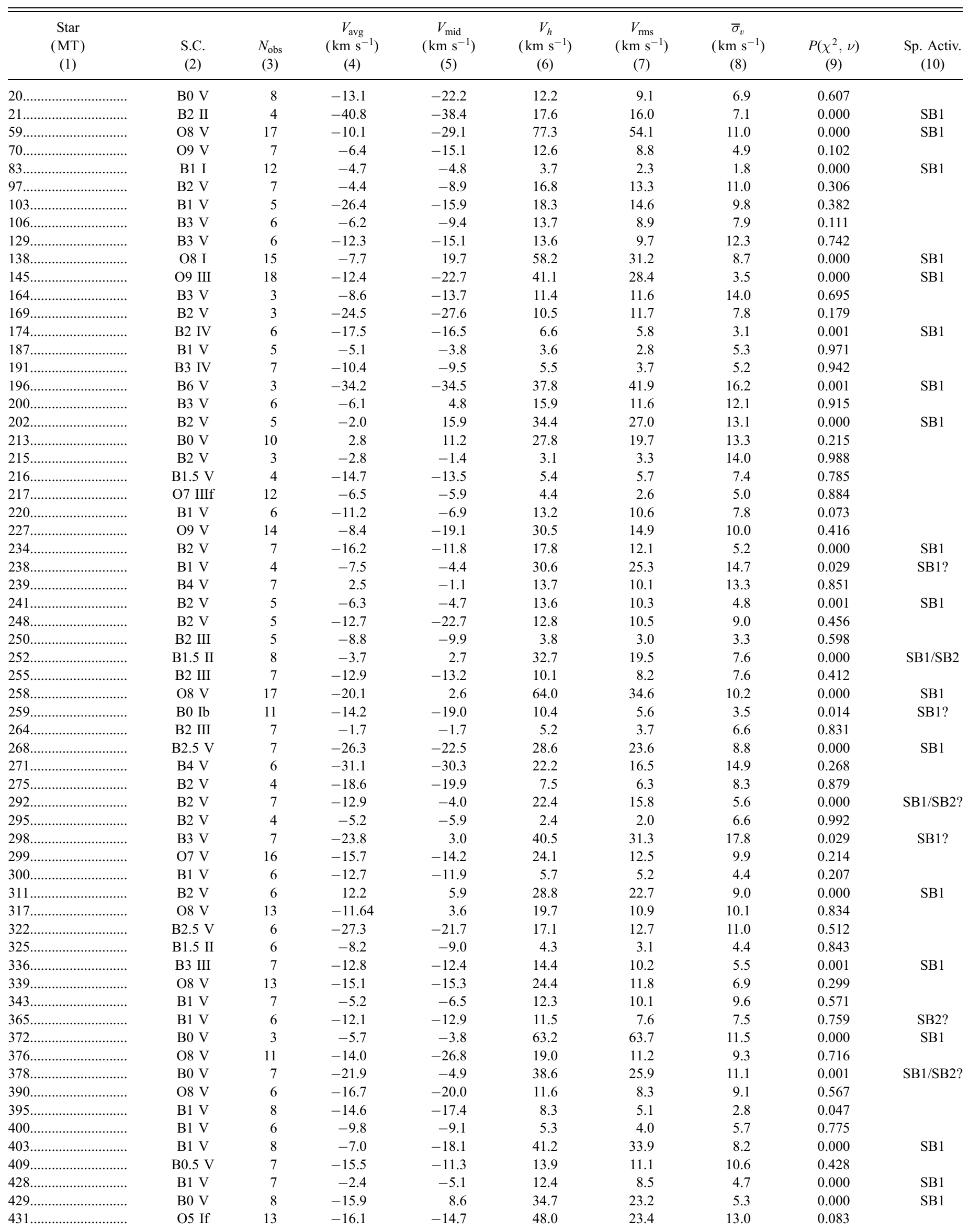


TABLE 5-Continued

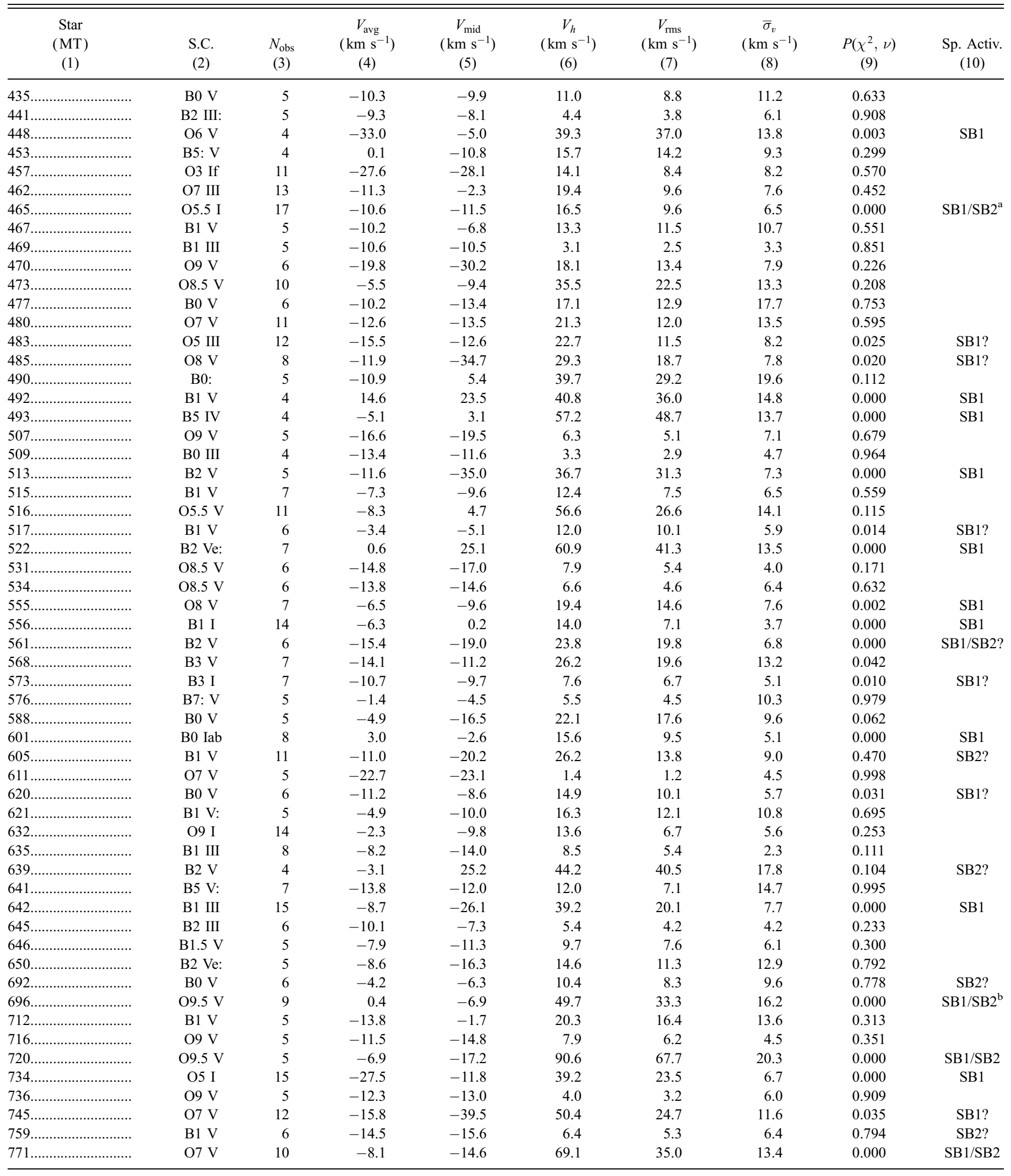

NotES._Col. (1): Star name in MT91 nomenclature. Col. (2): Spectral types as determined/accepted by this survey. Col. (3): Number of usable observations. Col. (4): The weighted average heliocentric velocity. Col. (5): $V_{\operatorname{mid}} \equiv 0.5\left(V_{\max }+V_{\min }\right)$, the simple average of the largest and smallest observed heliocentric velocity. Col. (6): $V_{h}=$ $0.5\left(V_{\max }-V_{\min }\right)$, a measure of the velocity semiamplitude. Col. (7): The rms heliocentric velocity dispersion of all observations. Col. (8): The mean velocity uncertainty, averaged over all observations. Col. (9): The probability $(P)$ that $\chi^{2}$ would be exceeded by chance, given $\nu=N_{\text {obs }}-1$ degrees of freedom. Col. (10): Spectroscopic activity, where "SB1" represents probable single-lined variability $\left[P\left(\chi^{2}, \nu\right) \leq 0.01\right]$, "SB1?" represents possible single-lined variability $\left[0.01<P\left(\chi^{2}, \nu\right) \leq 0.04\right]$, "SB2?" represents a possible double-lined binary signature, and "SB2" is a definite double-lined signature.

a Spectroscopic period of $P=21.908$ days (De Becker et al. 2004).

${ }^{\mathrm{b}}$ Photometric period of $P=1.46$ days (Rios \& DeGioia-Eastwood 2004). 


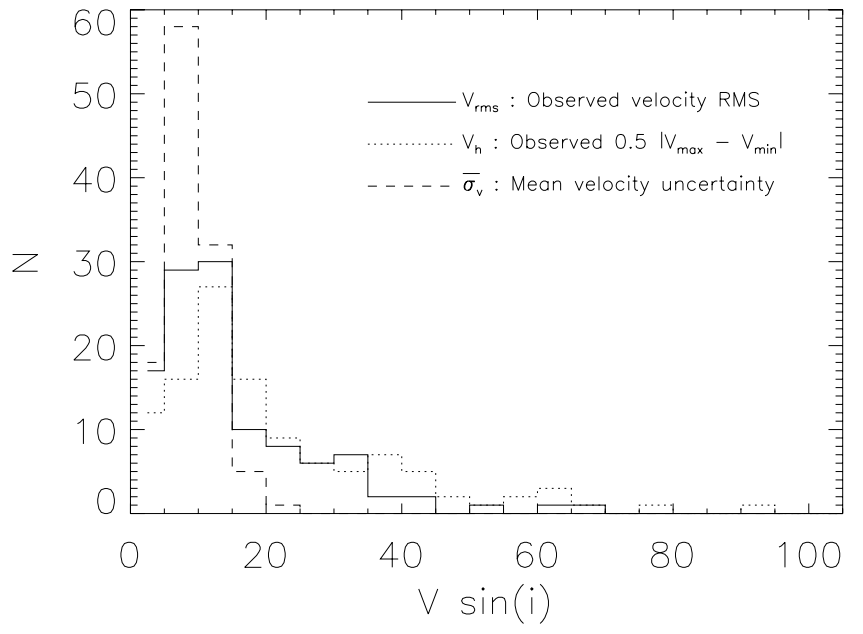

FIG. 11.-Distribution of observed velocity dispersions, $V_{\text {rms }}$ (solid line), velocity semiamplitudes, $V_{h} \equiv 0.5\left|V_{\max }-V_{\min }\right|$ (dotted line), and the mean velocity uncertainties (dashed line) for the sample.

scatter the data into higher velocity bins. The maximum observed semiamplitudes fall mostly between 10 and $40 \mathrm{~km} \mathrm{~s}^{-1}$, with a significant tail toward higher velocities out to $\sim 90 \mathrm{~km} \mathrm{~s}^{-1}$. The uncertainties lie in the characteristic range $5-15 \mathrm{~km} \mathrm{~s}^{-1}$.

The velocity results in Table 5 show a wide range of characteristics, including stars with large variations and stars with no significant variations. We used the method presented in Dequennoy \& Mayor (1991) to identify probable spectroscopic variables in our survey. In Figure 12 we show the distribution of probabilities that $\chi^{2}$ (as considered about the weighted mean of the measurements) would be exceeded given $\nu=N_{\text {obs }}-1$ degrees of freedom. As in Dequennoy \& Mayor (1991), the distribution is relatively flat except for values $P\left(\chi^{2}, \nu\right) \leq 0.01$. Column (9) of Table 5 lists the computed probabilities for the 120 stars examined. We identify in column (10) all stars with $P\left(\chi^{2}, \nu\right) \leq 0.01$ as probable SB1s (36 stars). In addition, we also identify the stars with $0.01<P\left(\chi^{2}, \nu\right)<0.04$ as possible SB1s (nine stars). The remaining objects are probable systems comprised of single stars, systems viewed at low inclinations, and systems with very low mass companions or very long periods.

In Figures $13-16$ we show heliocentric velocity versus time plots for four objects with minor or no velocity variations: MT

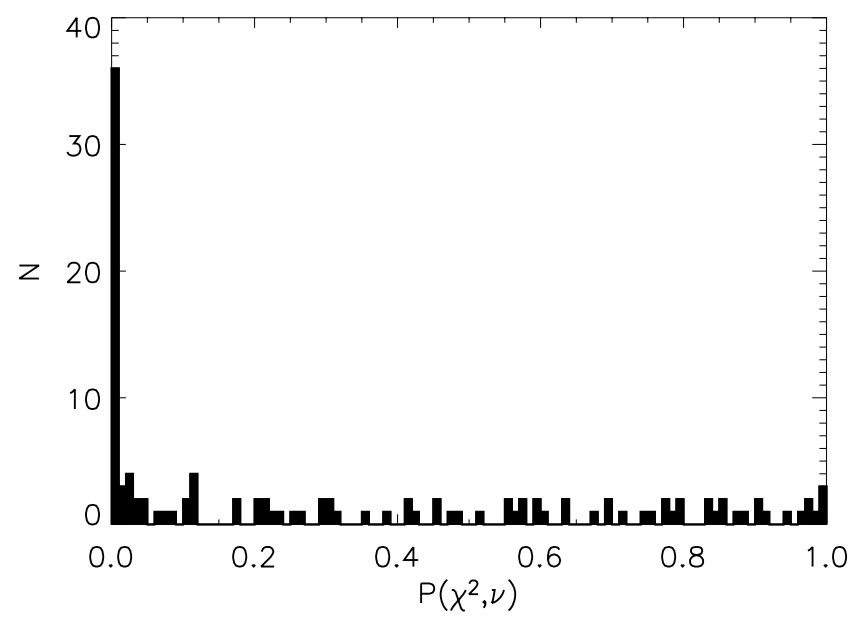

FIG. 12.-Distribution of probabilities that $\chi^{2}$ (as determined about the weighted mean) would be exceeded given $\nu=N_{\mathrm{obs}}-1$ degrees of freedom The discontinuity at $P\left(\chi^{2}, \nu\right)=0.01$ shows the change in the distribution from probable nonvariables to probable variables within the sample.

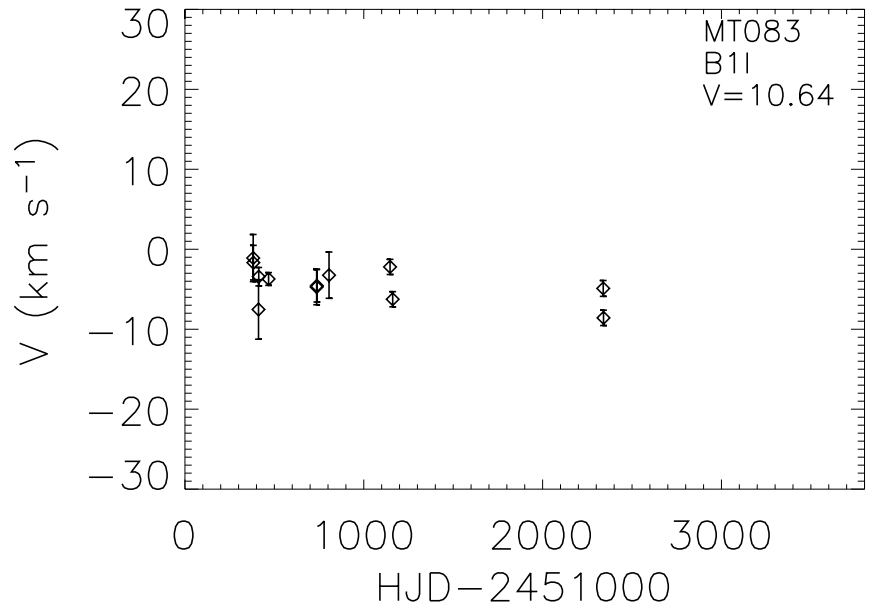

FIG. 13.-Relative radial velocity variation for MT 083 . MT 083 shows variations $\leq 5 \mathrm{~km} \mathrm{~s}^{-1}$ but is considered an SB1 since $P\left(\chi^{2}, \nu\right)<0.0004$.

083 (B1 I; SB1), MT 217 (O7 II), MT 264 (B2 II), and MT 317 $(\mathrm{O} 8 \mathrm{~V})$. The data for most objects cover a time interval of $\sim 5.5 \mathrm{yr}$ from 1999 July through 2004 November. The evolved systems among this group have some of the smallest velocity uncertainties in the survey $\left(<5 \mathrm{~km} \mathrm{~s}^{-1}\right)$ and are particularly well suited to assess the level of possible systematic velocity errors from epoch to epoch. MT 083 in particular (Fig. 13) exhibits no epoch-to-epoch variations greater than $\sim 5 \mathrm{~km} \mathrm{~s}^{-1}$ but has $P\left(\chi^{2}, \nu\right)<0.0004$, indicating that it is a low-amplitude spectroscopic variable. This system also exhibits some photometric variability, raising the possibility that the velocity variations may be attributable to atmospheric activity (Kukarkin \& Kholopov 1982). In comparison with the small velocity variations present among these four objects, the magnitude of the Doppler correction due to the Earth's orbital motion ranges from $\sim 8 \mathrm{~km} \mathrm{~s}^{-1}$ in July to roughly $-16 \mathrm{~km} \mathrm{~s}^{-1}$ in November. The constancy of the velocities, especially for evolved stars that have small uncertainties, among this subset of objects provides reassurance that systematic errors do not dominate the results.

Figures 17-22 show examples of some of the more prominent large-amplitude systems. In Figure 17 we present the velocity curve of MT 059 . This star exhibits large velocity variations of $\sim 100 \mathrm{~km} \mathrm{~s}^{-1}$ with an average uncertainty of $\sim 11 \mathrm{~km} \mathrm{~s}^{-1}$ on a timescale of $\sim 3$ days. Although our present data are not sufficiently time sampled to uniquely determine the orbital parameters,

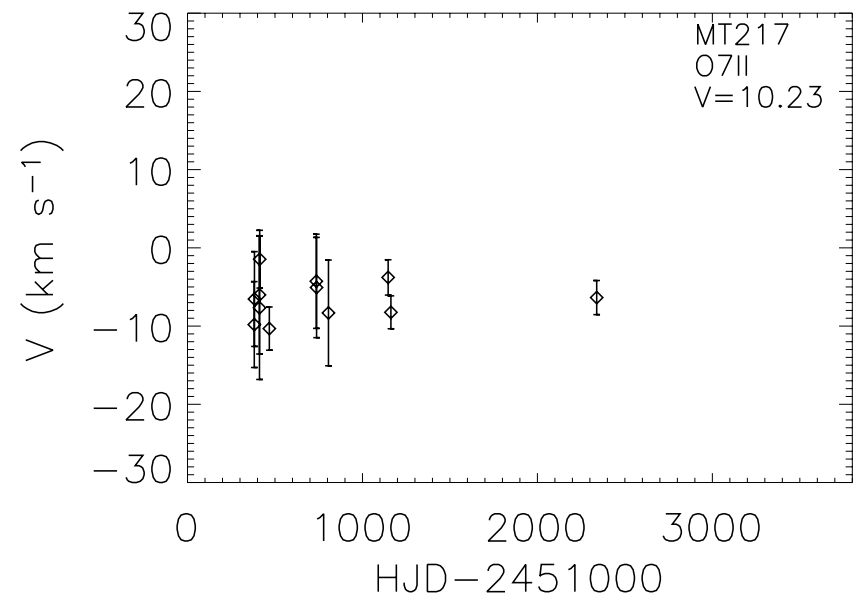

FIG. 14.- Relative radial velocity variation for MT 217, a system with little or no variation. 


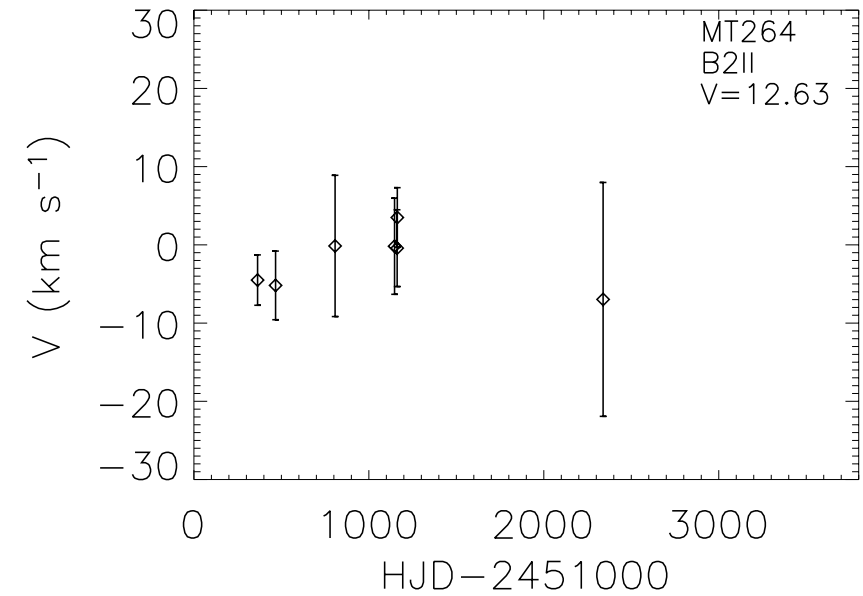

FIG. 15.- Relative radial velocity variation for MT 264, a system with little or no variation.

these rough values imply a secondary mass of $\geq 7 M_{\odot}$ and an orbital separation of $\sim 0.12 \mathrm{AU}$ assuming an inclination near $i=90^{\circ}$ and a circular orbit. Figure 18 shows MT 138, an O8 I star with an amplitude of nearly $85 \mathrm{~km} \mathrm{~s}^{-1}$, an average uncertainty of $\sim 9 \mathrm{~km} \mathrm{~s}^{-1}$, and variability on a several day timescale. These rough values imply a companion mass of $\geq 8 M_{\odot}$ and an orbital separation of $\sim 0.17$ AU assuming an inclination near $i=90^{\circ}$. Figure 19 shows MT 145, another evolved star (O9 III). It has an amplitude of nearly $50 \mathrm{~km} \mathrm{~s}^{-1}$ and an average uncertainty of $\sim 4 \mathrm{~km} \mathrm{~s}^{-1}$ with an apparent period of several days. The implied companion parameters are $M \geq 5 M_{\odot}$ and $a \simeq 0.15 \mathrm{AU}$. Another O8 V star (MT 258) is presented in Figure 20. It displays an amplitude of at least $45 \mathrm{~km} \mathrm{~s}^{-1}$ and an average uncertainty of $\sim 10 \mathrm{~km} \mathrm{~s}^{-1}$ with a period of several days. The implied companion parameters are $M \sim 3 M_{\odot}$ and $a \simeq 0.12$ AU. MT 492, a B1 V shown in Figure 21, is representative of early B stars that have relatively few observations but still strong evidence for velocity variability. It has an amplitude of at least $50 \mathrm{~km} \mathrm{~s}^{-1}$ and an average uncertainty of $\sim 15 \mathrm{~km} \mathrm{~s}^{-1}$. MT 734, shown in Figure 22 , is one of the brightest and most massive stars in the survey (O5 I). We estimate an amplitude of $\sim 40 \mathrm{~km} \mathrm{~s}^{-1}$, an average uncertainty of $\sim 7 \mathrm{~km} \mathrm{~s}^{-1}$, and variations consistent with a period on the order of days. The implied companion parameters are $M \geq 4 M_{\odot}$ and $a \simeq 0.15 \mathrm{AU}$. At this time we can only make rough estimates of the orbital parameters for these few objects

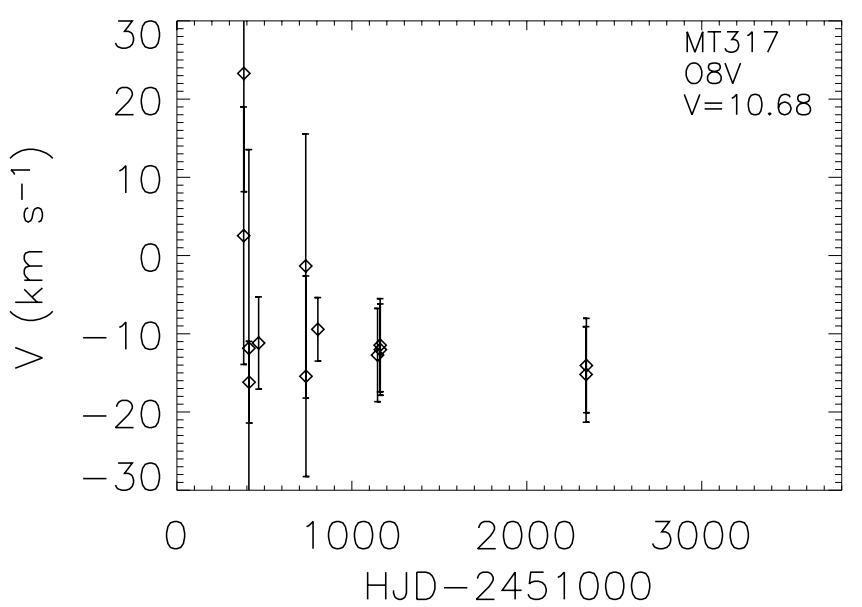

FIG. 16.- Relative radial velocity variation for MT 317, a system with little or no variation.

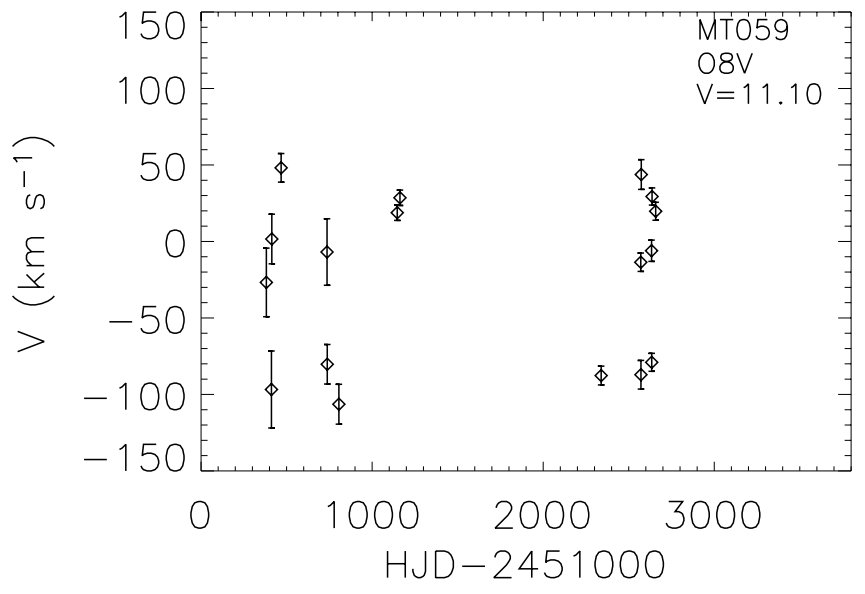

FIG. 17.- Relative radial velocity variation for MT 059 , one of the more prominent velocity-variable systems. MT 059 is among the 36 stars with $P\left(\chi^{2}, \nu\right) \leq 0.01$.

with the largest velocity amplitudes. Our present data, however, do not allow us to rule out short-period aliases or nonperiodic variations.

\subsection{Double-lined Spectroscopic Binaries}

The cross-correlation analyses reveal five systems with doublelined spectra and eight additional systems with possible doublelined signatures (three of these are also designated SB1). These two groups are designated in column (10) of Table 5 as SB2 and SB2?, respectively. The latter group contains objects with variable cross-correlation widths, indicating possible multiplicity where the stellar features are blended at the resolution of the spectra. The former group contains objects where the velocity separation of the features is sufficient to allow the recognition of distinct spectral components at one or more epochs. Two of these systems are previously identified as SB2 in the literature: MT 465 (De Becker et al. 2004) and MT 696 (Rios \& DeGioiaEastwood 2004).

MT 252 shows two velocity components in the $\mathrm{He}$ I and $\mathrm{Mg}$ I lines and variable width and asymmetry in the hydrogen lines during two epochs (2001 September 8-9). The 2001 September 8 spectrum displays a maximum velocity amplitude of $101 \mathrm{~km} \mathrm{~s}^{-1}$ (He I) for both components, relative to the mean systemic velocity. This implies a mass ratio near unity. The ratio of luminosities of the two components is also near unity. Given the B1.5 III

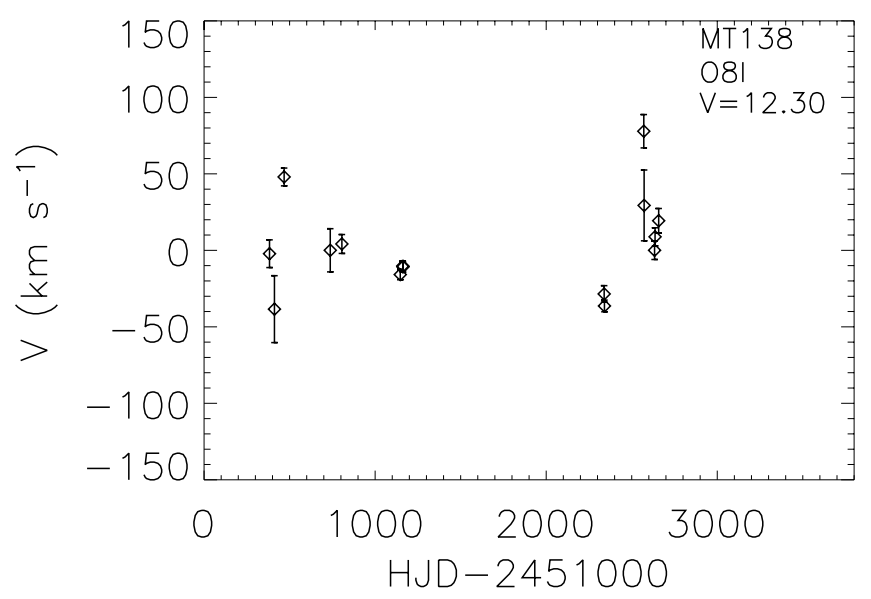

FIG. 18.- Relative radial velocity variation for MT 138, one of the more prominent velocity-variable systems. 


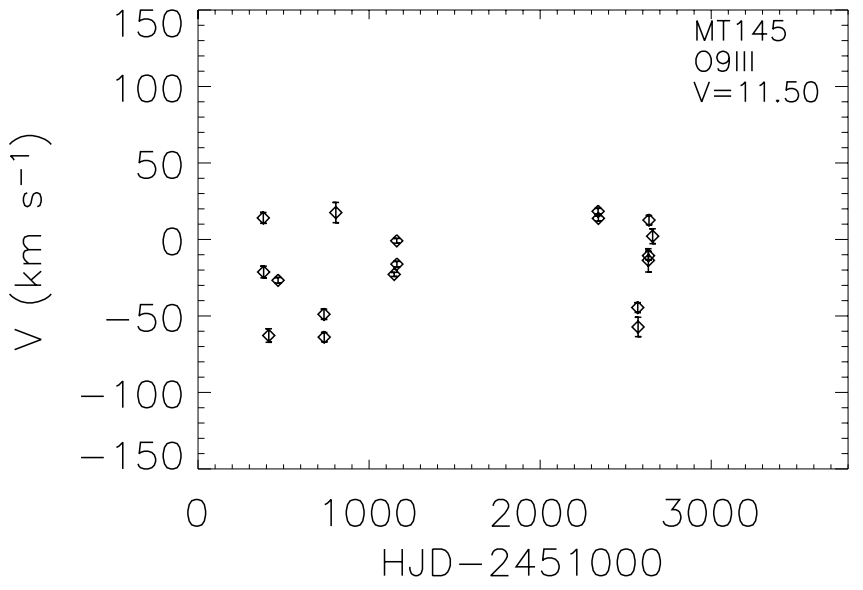

FIG. 19.- Relative radial velocity variation for MT 145, one of the more prominent velocity-variable systems.

spectral classification, this suggests that the secondary is probably another B1.5 III or possibly an O9 V.

MT 465 (Cyg OB2 No. 8a) is reported to be a binary consisting of an O6 and an O5.5 star (De Becker et al. 2004). Despite the obvious double-lined spectra, 23 day period, and $200 \mathrm{~km} \mathrm{~s}^{-1}$ velocity separation reported in that work, the components are blended in all of our spectra. We therefore report this star as an SB1 and include an SB2 designation only because of the De Becker et al. (2004) study.

MT 696, an $09.5 \mathrm{~V}$, is reported to be a double system containing an early B secondary (Rios \& DeGioia-Eastwood 2004). At least four of our observations of this star displayed dual velocity components in multiple lines. The ratio of velocity amplitudes is $\sim 0.7$ with a maximum velocity separation of $540 \mathrm{~km} \mathrm{~s}^{-1}$ on 2005 September 22 . With a mass $70 \%$ of the $09.5 \mathrm{~V}$ primary, the deduced secondary spectral type (B1/B2 V) agrees with the findings of Rios \& DeGioia-Eastwood (2004).

MT 720, classified as an $09.5 \mathrm{~V}$, shows multiple velocity components, indicating that it is a probable triple system. The 2004 November 30 spectrum displays two velocity features with a separation of $350 \mathrm{~km} \mathrm{~s}^{-1}$ for the He I lines. The 2001 August 24 spectrum also shows two velocity components, but there is an additional asymmetry on the redshifted side of the $\mathrm{H}$ and $\mathrm{He}$ features. The 2001 September 9 spectrum clearly shows at least three velocity components in multiple lines. Luminosities among the primary, secondary, and tertiary are near unity and suggest

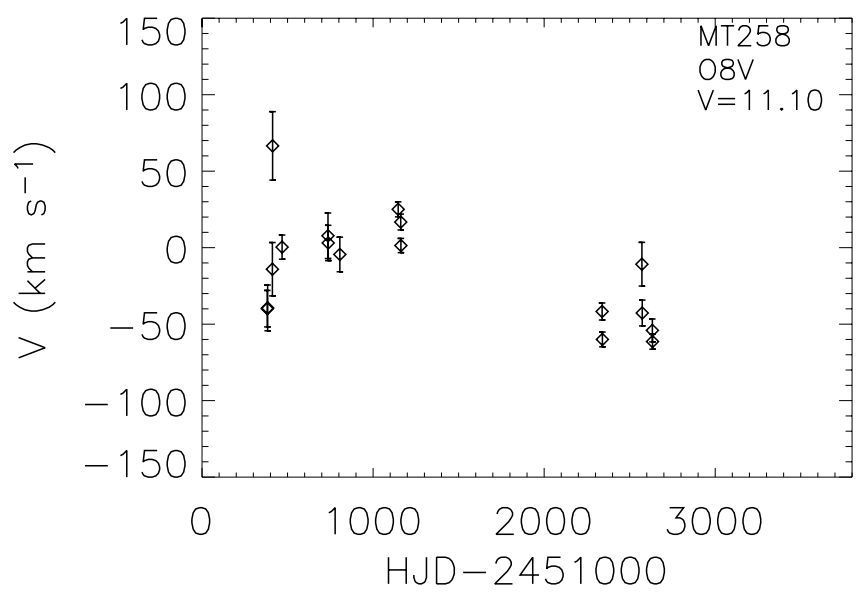

FIG. 20.- Relative radial velocity variation for MT 258, one of the more prominent velocity-variable systems.

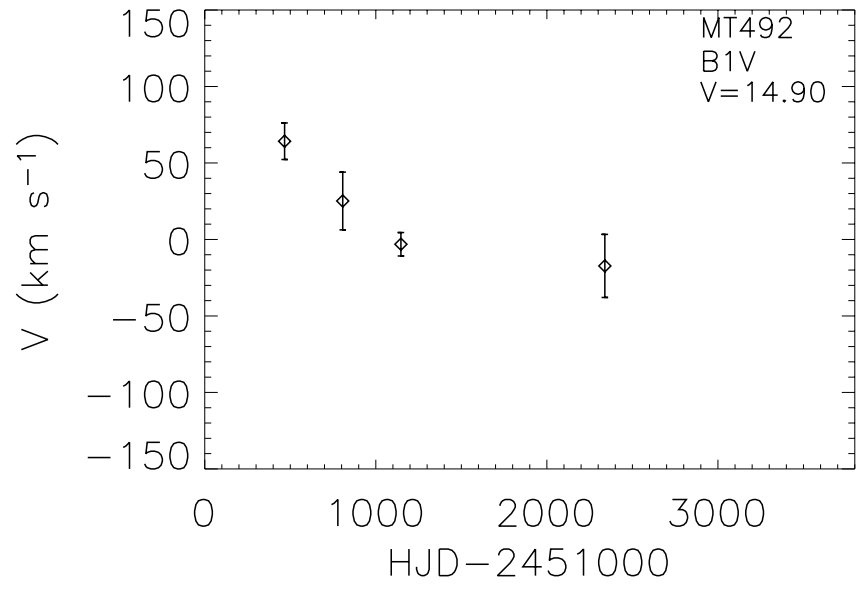

FIG. 21.- Relative radial velocity variation for MT 492, one of the more prominent velocity-variable systems.

comparable masses indicative of a system with three late-type $\mathrm{O}$ stars or B0 stars.

MT 771, an $\mathrm{O} 7 \mathrm{~V}$, shows He I double-lined components in three epochs (2001 September 9 and 2004 November 28-29), with a maximum velocity separation of $275 \mathrm{~km} \mathrm{~s}^{-1}$. The ratio of velocity amplitudes is greater than 0.8 as measured about the systemic velocity calculated in the 2004 November 30 spectrum. The ratio of luminosities is $\sim 0.75$, suggesting that the companion is a late $\mathrm{O}$ star.

\subsection{Velocity Dispersion of $\mathrm{Cyg} O B 2$}

Figure 23 provides histograms of systemic velocities for our two methods of velocity measurement. The shaded histogram represents the $V_{\text {mid }}$ velocities in column (5) of Table 5. The open histogram represents the $V_{\text {avg }}$ velocities from column (4) of Table 5. A Gaussian fit to the data yields an FWHM of $8.01 \pm 0.26 \mathrm{~km} \mathrm{~s}^{-1}$ for the shaded histogram and $5.7 \pm 0.17 \mathrm{~km} \mathrm{~s}^{-1}$ for the open histogram. This translates to a one-dimensional radial velocity dispersion of $\sigma_{V}=3.41 \pm 0.11 \mathrm{~km} \mathrm{~s}^{-1}$ and $2.44 \pm 0.07 \mathrm{~km} \mathrm{~s}^{-1}$ for $V_{\text {mid }}$ and $V_{\text {avg }}$, respectively. Both fits show that the mean systemic velocity of Cyg OB2 stars is $\bar{V}_{\text {hel }}=-10.3 \pm 0.3 \mathrm{~km} \mathrm{~s}^{-1}$. Because the $V_{\text {mid }}$ values have more outliers at large relative velocities, this measurement is best regarded as an upper limit to the true velocity dispersion. By comparison, typical open cluster velocity dispersions lie in the range $1-2.5 \mathrm{~km} \mathrm{~s}^{-1}$ and may depend on initial conditions (Bate $\&$ Bonnell 2005). The velocity

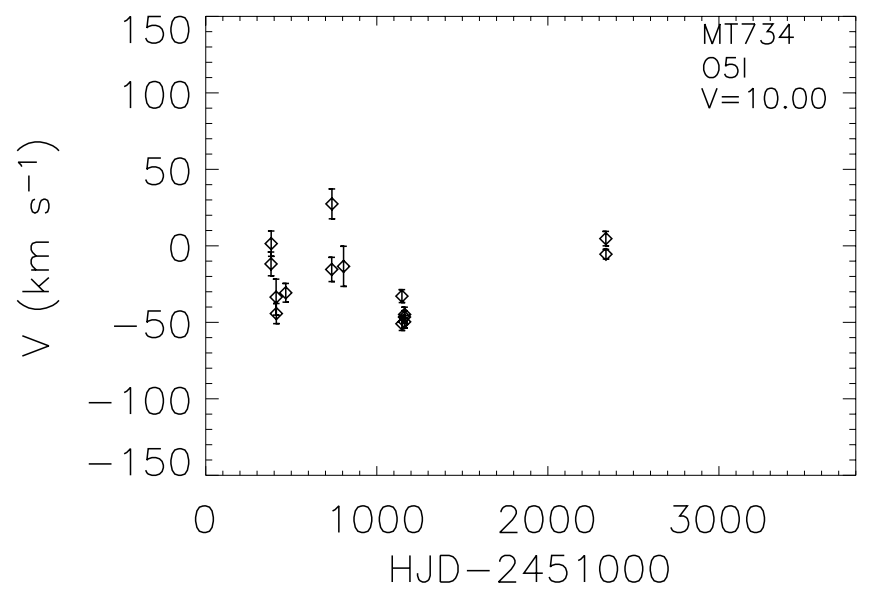

FIG. 22.- Relative radial velocity variation for MT 734, one of the more prominent velocity-variable systems. 


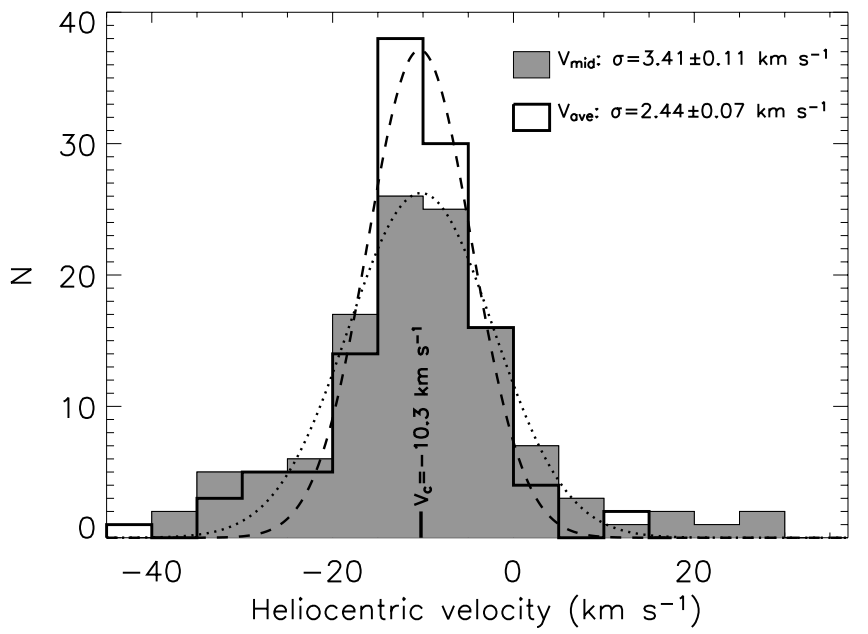

Fig. 23.-Mean systemic velocity distribution for Cyg OB2 stars listed in Table 5. The open histogram represents the $V_{\text {avg }}$ velocities in col. (4). The shaded histogram represents the $V_{\text {mid }}$ velocities from col. (5). Gaussian fits to each histogram yield a mean systemic velocity of roughly $-10 \mathrm{~km} \mathrm{~s}^{-1}$ and dispersions of $\sigma=2.44 \pm 0.07 \mathrm{~km} \mathrm{~s}^{-1}$ for $V_{\text {avg }}$ and $\sigma=3.41 \pm 0.11 \mathrm{~km} \mathrm{~s}^{-1}$ for $V_{\text {mid. }}$.

dispersions of other open clusters, such as the Orion Nebula cluster (Jones \& Walker 1988; $2 \mathrm{~km} \mathrm{~s}^{-1}$ ), Perseus OB2 (Steenbrugge et al. 2003; 1-3 $\mathrm{km} \mathrm{s}^{-1}$ ), or Scorpius OB2 (de Bruijne 1999; $1.0-1.5 \mathrm{~km} \mathrm{~s}^{-1}$ ), are consistent with our measurement of $V_{\text {avg }}=$ $2.44 \pm 0.07 \mathrm{~km} \mathrm{~s}^{-1}$. We also examined systemic velocity as a function of position within the cluster and found no evidence of large-scale velocity patterns that might indicate the presence of kinematic subgroups.

\subsection{Runaways in Cyg OB2}

Nearly $20 \%$ of all O stars are runaways, with the other $70 \%-$ $80 \%$ locked in open clusters or OB associations (Kaper et al. 1999; Gies 1987). The origin of runaway stars is still uncertain, but the two leading theories, asymmetric supernova ejection and dynamical interaction, require the existence of a companion (Blaauw 1961, 1993). With more than $30 \mathrm{O}$ stars listed in Table 5 and a binary fraction of at least $50 \%$ among the early-type stars (Garcia \& Mermilliod 2001; Gies 1987), one might expect to see two to three OB runaways in Cyg OB2. Runaways are usually defined as having space velocities exceeding $30-40 \mathrm{~km} \mathrm{~s}^{-1}$ (Blaauw 1961) relative to their parent cluster or mean local Galactic rotation velocity. Even at minimal runaway speeds, the association crossing time is $\leq 0.5 \mathrm{Myr}$ for a diameter of $\sim 30 \mathrm{pc}$. Thus, any runaways with velocities that are primarily tangential to the line of sight would travel well beyond the canonical boundaries of Cyg OB2 within its lifetime. Comerón et al. (1998) speculate that some of the OB stars in the Cygnus region that have high proper motions may have been ejected from Cyg OB2. Most Cyg OB2 members lack proper-motion measurements, and our data are sensitive only to motions in the radial direction. Therefore, we would expect, at most, one to two runaway stars with large radial velocity components detectable in this survey.
Figure 23 shows that no stars have radial velocities larger than $35 \mathrm{~km} \mathrm{~s}^{-1}$ relative to the mean systemic velocity of the association. The most notable outliers, which have relative velocities of $20-30 \mathrm{~km} \mathrm{~s}^{-1}$, are also those with the fewest measurements and, therefore, are the most uncertain. We detect no strong candidates for runaway stars. Therefore, we conclude that there is little or no evidence for OB runaways in the radial direction and likely very few runaways within a $\sim 30^{\prime}$ radius of the cluster center.

\section{CONCLUSIONS}

We conducted a radial velocity survey of the Cyg OB2 association over a $6 \mathrm{yr}$ time interval to search for MCBs using spectroscopic data from the Keck, Lick, WIYN, and WIRO observatories. We obtained 1139 spectra to measure radial velocities and radial velocity variations on 146 OB stars. There were 73 identified as new early types. The calculated mean distance modulus for Cyg OB2 stars is $\sim 11.3 \mathrm{mag}$, which is in good agreement with previous estimates. Of the 146 total OB stars, analysis of the 143 provisional members yielded an IMF slope of $\Gamma=-2.2 \pm 0.1$. There were a number of minor spectral classification differences, including Cyg OB2 No. 12 and No. 21. No. 12 showed evidence of a B3 Iae spectral classification in at least one epoch and a temperature class variation (B3-B8) over $1 \mathrm{yr}$. We utilized two methods for determining velocity variations, including Gaussian profile fitting and crosscorrelation techniques. Both methods yielded similar results, where 36 stars had a probability of $P\left(\chi^{2}, \nu\right) \leq 0.01$ and nine stars had a probability of $0.01<P\left(\chi^{2}, \nu\right) \leq 0.04$. In addition, we detected five SB2 systems and eight possible SB2 systems (three of which were also designated SB1). This translates to a lower limit on the massive binary frequency of $30 \%$ (36 out of 120 stars) to $42 \%$ (50 out of 120 stars). The calculated velocity dispersion for Cyg OB2 is $2.44 \pm 0.07 \mathrm{~km} \mathrm{~s}^{-1}$, which is typical of open clusters, and despite the richness of the association and the number of stars surveyed, we detected no obvious OB runaways.

We thank the time allocation committees of the Lick, Keck, WIYN, and WIRO observatories for granting us observing time and making this project possible. This paper has been greatly improved by the detailed review and suggestions from an anonymous referee. We are grateful for support from the National Science Foundation through the Research Experiences for Undergraduates (REU) program grant AST 03-53760 and through grant AST 03-07778, as well as the support of the Wyoming NASA Space Grant Consortium. The work of C. F. was in part under the auspices of the US Department of Energy and supported by its contract W-7405-ENG-36 to Los Alamos National Laboratory and by the National Science Foundation under grant PHY99-07949.

Facilities: WIRO, WIYN, Shane, Keck:I
Abbott, D. C., Bieging, J. H., \& Churchwell, E. 1981, ApJ, 250, 645

Bate, M. R., \& Bonnell, I. A. 2005, MNRAS, 356, 1201

Benaglia, P., Romero, G. E., Stevens, I. R., \& Torres, D. F. 2001, A\&A, 366, 605

Bieging, J. H., Abbott, D. C., \& Churchwell, E. 1989, ApJ, 340, 518

Blaauw, A. 1961, Bull. Astron. Inst. Netherlands, 15, 265

. 1993, Irish Astron. J., 16, 141

Bohannan, B., \& Conti, P. S. 1976, ApJ, 204, 797

\section{REFERENCES}

Bonnell, I. A., Bate, M. R., \& Zinnecker, H. 1998, MNRAS, 298, 93

Comerón, F., Torra, J., \& Gomez, A. E. 1998, A\&A, 330, 975

Comerón, F., et al. 2002, A\&A, 389, 874

Cowley, C., Wiese, W. L., Fuhr, J., \& Kuznetsova, L. A. 2000, in Astrophysical

Quantities, ed. A. N. Cox (4th ed.; New York: Springer), 53

De Becker, M., Rauw, G., \& Manfroid, J. 2004, A\&A, 424, L39

de Bruijne, J. H. 1999, MNRAS, 310, 585

Dequennoy, A., \& Mayor, M. 1991, A\&A, 248, 485 
Drilling, J. S., \& Landolt, A. U. 2000, in Astrophysical Quantities, ed. A. N. Cox (4th ed.; New York: Springer), 381

Fryer, C. L., Burrows, A., \& Benz, W. 1998, ApJ, 496, 333

Fryer, C. L., Woosley, S. E., \& Hartmann, D. H. 1999, ApJ, 526, 152

Fullerton, A. W., Gies, D. R., \& Bolton, C. T. 1996, ApJS, 103, 475

Garcia, B., \& Mermilliod, J. C. 2001, A\&A, 368, 122

Garmany, C. D., Conti, P. S., \& Massey, P. 1980, ApJ, 242, 1063

Gies, D. R. 1987, ApJS, 64, 545

Gottleib, E. W., \& Liller, W. 1978, ApJ, 225, 488

Hall, D. S. 1974, Acta Astron., 24, 69

Hanson, M. M. 2003, ApJ, 597, 957

Harnden, F. R., et al. 1979, ApJ, 234, L51

Hillwig, T. C., Gies, D. R., Baynuolo, W. G., Huang, W., McSwain, M. V., \& Wingert, D. W. 2006, ApJ, 639, 1069

Humphreys, R. M., \& McElroy, D. B. 1984, ApJ, 284, 565

Johnson, H. L. 1968, in Nebulae and Interstellar Matter, ed. B. M. Middlehurst \& L. H. Aller (Chicago: Univ. Chicago Press), 167

Johnson, H. L., \& Morgan, W. W. 1954, ApJ, 119, 344

Jones, B. F., \& Walker, M. F. 1988, AJ, 95, 1755

Kaper, L., Comerón, F., \& Barziv, O. 1999, in IAU Symp. 193, Wolf-Rayet Phenomena in Massive Stars and Starburst Galaxies, ed. K. A. van der Hucht, G. Koenigsberger, \& P. R. J. Eenens (San Francisco: ASP), 316

Kazarovets, E. V., Samus, N. N., \& Durlevich, O. V. 2000, Inf. Bull. Variable Stars, 4870, 1

Knödlseder, J. 2000, A\&A, 360, 539 (K00)

Kukarkin, B. V., \& Kholopov, P. N. 1982, New Catalogue of Suspected Variable Stars (Moscow: Nauka)

Kurtz, M. J., Mink, D. J., Wyatt, W. F., Fabricant, D. G., Torres, G., Kriss, G. A., \& Tonry, J. L. 1992, in ASP Conf. Ser. 25, Astronomical Data Analysis Software and Systems I, ed. D. M. Worrall, C. Biemesderfer, \& J. Barnes (San Francisco: ASP), 432

Lanz, T., \& Hubeny, I. 2003, ApJS, 146, 417

Leitherer, C., \& Wolf, B. 1982, Mitt. Astron. Ges. Hamburg, 55, 55

Lejeune, T., \& Schaerer, D. 2001, A\&A, 366, 538

Lennon, D. J., Dufton, P. L., \& Fitzsimmons, A. 1992, A\&AS, 94, 569

Leung, K.-C., \& Schneider, D. P. 1978, ApJ, 224, 565

Maeder, A., \& Meynet, G. 1988, A\&AS, 76, 411

Martins, F., Schaerer, D., \& Hillier, D. J. 2005, A\&A, 436, 1049

Massey, P., Johnson, K., \& DeGioia-Eastwood, K. 1995, ApJ, 454, 151
Massey, P., \& Thompson, A. B. 1991, AJ, 101, 1408 (MT91)

Nations, H. L., \& Pierce, M. J. 2002, BAAS, 34, 749

Patriarchi, P., Morbidelli, L., \& Perinotto, M. 2003, A\&A, 410, 905

Penrod, G. D. 1986, PASP, 98,35

Pigulski, A., \& Kolaczkowski, Z. 1998, MNRAS, 298, 753

Press, W. H., Teukolsky, S. A., Vetterling, W. T., \& Flannery, B. P. 1992,

Numerical Recipes in C (Cambridge: Cambridge Univ. Press)

Rios, L. Y., \& DeGioia-Eastwood, K. 2004, BAAS, 36, 1348

Romano, G. 1969, Mem. Soc. Astron. Italiana, 40, 375

Salpeter, E. E. 1955, ApJ, 121, 161

Schulte, D. H. 1958, ApJ, 128, 41

Setia Gunawan, D. Y. A., de Bruyn, A. G., van der Hucht, K. A., \& Williams, P. M. 2003, ApJS, 149, 123

Sharpless, S. 1957, PASP, 69, 239

Skrutski, M. F., et al. 2006, AJ, 131, 1163

Snow, T., Zukowski, D., \& Massey, P. 2002, ApJ, 578, 877

Souza, S. P., \& Lutz, B. L. 1980, ApJ, 235, L87

Steenbrugge, K. C., de Bruijne, J. H., Hoogerwerf, R., \& de Zeeuw, P. T. 2003 , A\&A, 402, 587

Torres-Dodgen, A. V., Tapia, M., \& Carroll, M. 1991, MNRAS, 249, 1

Vanbeveren, D. 2004, EAS, 13, 141

Vanbeveren, D., De Loore, C., \& Van Rensbergen, W. 1998, A\&A Rev., 9, 63

van der Woerd, H., \& van den Heuvel, E. P. J. 1984, A\&A, 132, 361

Voelcker, K. 1975, A\&AS, 22, 1

Vogt, S. S., \& Penrod, G. D. 1983, ApJ, 275, 661

Vogt, S. S., et al. 1994, Proc. SPIE, 2198, 362

Walborn, N. R. 1973, ApJ, 180, L35

Walborn, N. R., \& Fitzpatrick, E. L. 1990, PASP, 102, 379

Waldron, W. L., Cassinelli, J. P., Miller, N. A., MacFarlane, J. J., \& Reiter, J. C. 2004, ApJ, 616, 542

Waldron, W. L., Corcoran, M. F., Drake, S. A., \& Smale, A. P. 1998, ApJS, 118,217

Wang, J., \& Zhu, Z. 2003, Chinese Phys. Lett., 20, 778

Wegner, W. 1994, MNRAS, 270, 229

Yamashita, Y., \& Nariai, K. 1977, An Atlas of Representative Stellar Spectra (Tokyo: Univ. Tokyo Press)

Zinnecker, H. 2003, in IAU Symp. 212, A Massive Star Odyssey: From Main Sequence to Supernova, ed. K. van der Hucht, A. Herrero, \& C. Esteban (San Francisco: ASP), 80 